\title{
Feuilletages et Transformations Périodiques
}

\author{
Dominique Cerveau and Julie Déserti
}

\section{CONTENTS}

1. Introduction

2. Quelques Définitions et Notations

3. Feuilletages Quadratiques et Involutions Birationnelles

4. Feuilletages de Degré Supérieur et Involutions

5. Feuilletages de Degré 3 et Trivolutions

6. Conclusion

Remerciements

Références
2000 AMS Subject Classification: 14E07, 37F75

Keywords: Foliation, birational involution
Bertini classified the birational involutions of the complex projective plane, but his geometric approach does not allow one easily to exhibit these maps explicitly. In this article, we present an effective approach to this problem by associating to each quadratic foliation a birational involution that is, in the generic case, a Geiser involution; this subject has already been covered by Geiser, Milinowski, Williams, and others. We end by conducting some experiments, from which we obtain trivolutions from some foliations of degree 3 .

La classification des involutions birationnelles du plan projectif complexe remonte essentiellement à Bertini ; cette classification de nature géométrique permet difficilement la construction explicite de telles involutions. Nous proposons une approche effective permettant d'associer à tout feuilletage quadratique une involution qui, dans le cas générique, est de Geiser; ce sujet a déjà été étudié par Geiser, Milinowski, Williams et alt. Nous présentons ensuite quelques expériences qui produisent des trivolutions à partir de feuilletages cubiques très spéciaux.

\section{INTRODUCTION}

Dans ce texte on va donner une construction qui relie feuilletages de degré 2 (resp. 3) et involutions (resp. trivolutions) birationnelles. À un feuilletage $\mathcal{F}$ de degré 2 du plan projectif complexe, on peut associer une involution birationnelle : une droite générique de $\mathbb{P}^{2}(\mathbb{C})$ a deux points de tangence avec $\mathcal{F}$; l'application $\mathcal{I}_{\mathcal{F}}$ qui permute ces deux points est une involution birationnelle dite involution associée à $\mathcal{F}$. On relie les points éclatés par $\mathcal{I}_{\mathcal{F}}$ et les points singuliers de $\mathcal{F}$; les courbes contractées par $\mathcal{I}_{\mathcal{F}}$ et les courbes des points de tangence entre $\mathcal{F}$ et les pinceaux de droites passant par les points singuliers de $\mathcal{F}$; l'adhérence des points d'inflexion de $\mathcal{F}$ et l'adhérence des points fixes de $\mathcal{I}_{\mathcal{F}}$. On montre que l'involution associée à un feuilletage quadratique générique de $\mathbb{P}^{2}(\mathbb{C})$ est une involution de Geiser; ceci permet d'en donner des exemples

(c) A K Peters, Ltd. 1058-6458/2010 $\$ 0.50$ per page Experimental Mathematics 19:3, page 447 
explicites. On s'intéresse en particulier au feuilletage $\mathcal{F}_{J}$ de Jouanolou de degré 2 pour lequel l'involution associée $\mathcal{I}_{\mathcal{F}_{J}}$ est une involution de Geiser; le groupe engendré par $\mathcal{I}_{\mathcal{F}_{J}}$ et le groupe d'isotropie de $\mathcal{F}_{J}$ est un sousgroupe fini d'ordre 42 , non linéarisable du groupe de Cremona. Traditionnellement les involutions de Geiser sont construites via la donnée d'un pinceau de cubiques en position générale (§3.2). Comme nous l'a indiqué le referee la construction explicite de telles involutions, ou les problèmes qui lui sont connexes, a intéressé de nombreux mathématiciens parmi lesquels il faut citer Weddle, Hart, Hesse, Chasles, Cayley, Geiser, Milinowski. Dans un article écrit en 1920 Williams redécouvre et précise la démarche de la construction de l'involution de Geiser associée à sept points en position générale [Williams 20]; il en donne les dégénérescences, dégénérescences associées à celles de la position des points. Évidemment la lecture de cet article montre l'étendue des connaissances sur le sujet mais présente une certaine difficulté due en particulier à l'évolution du langage et aux évidences propres à une époque dans un domaine qui revient à l'ordre du jour.

Réciproquement à une involution birationnelle $\mathcal{I}=$ $\left(\mathcal{I}_{1}, \mathcal{I}_{2}\right)$ on peut associer le feuilletage $\mathcal{F}$ décrit en carte affine par le champ de vecteurs

$$
\left(x-\mathcal{I}_{1}(x, y)\right) \frac{\partial}{\partial x}+\left(y-\mathcal{I}_{2}(x, y)\right) \frac{\partial}{\partial y} .
$$

Ce feuilletage est de degré pair $2 n$ avec $n>1$ et chaque droite générique contient $n$ orbites distinctes suivant $\mathcal{I}$; c'est par exemple le cas pour les involutions de Bertini où les orbites sont arrangées en constellations de 4 orbites en alignement. En degré 2 l'adhérence des points d'inflexion de $\mathcal{F}$ est contenue dans l'adhérence des points fixes de $\mathcal{I}_{\mathcal{F}}$; on verra que ce n'est plus le cas en degré supérieur : les courbes de points d'inflexion peuvent être permutées $\operatorname{par} \mathcal{I}_{\mathcal{F}}$.

Considérons un feuilletage $\mathcal{F}$ de degré 3 sur $\mathbb{P}^{2}(\mathbb{C})$. Toute droite générique de $\mathbb{P}^{2}(\mathbb{C})$ est tangente à $\mathcal{F}$ en trois points. L' « application» qui échange ces trois points est en général multivaluée; on donne un critère qui assure que cette application est birationnelle ce qui permet de produire des exemples explicites. On considère en particulier le cas des feuilletages homogènes génériques; l'éventuelle trivolution associée est nécessairement de Jonquières. On produit des exemples qui permettent de construire des 3-tissus hexagonaux $\operatorname{sur} \mathbb{C}^{2}$.

\section{QUELQUES DÉFINITIONS ET NOTATIONS}

\subsection{Transformations Birationelles}

Une transformation rationnelle $f: \mathbb{P}^{2}(\mathbb{C}) \rightarrow \mathbb{P}^{2}(\mathbb{C})$ du plan projectif complexe dans lui-même est de la forme

$$
(x: y: z) \mapsto\left(f_{0}(x, y, z): f_{1}(x, y, z): f_{2}(x, y, z)\right),
$$

les $f_{i}$ désignant des polynômes homogènes de même degré sans facteur commun. Le degré de $f$ est, par définition, le degré des $f_{i}$. Une transformation birationnelle est une transformation rationnelle qui admet un inverse, luimême rationnel. Le groupe de Cremona, noté $\operatorname{Bir}\left(\mathbb{P}^{2}(\mathbb{C})\right.$ ), est le groupe des transformations birationnelles du plan projectif complexe. Le lieu d'indétermination de $f$, ou encore l'ensemble des points éclatés par $f$, est le lieu d'annulation des $f_{i}$; on le désigne par $\operatorname{Ind}(f)$. Le lieu exceptionnel de $f$ est l'ensemble des zéros du déterminant jacobien de $f$; on le note $\operatorname{Exc}(f)$. On dit que les éléments de $\operatorname{Exc}(f)$ sont les courbes contractées par $f$. Désignons par Fix $(f)$ l'adhérence des points fixes de $f$; c'est une union de courbes et de points isolés.

Exemple 2.1. La transformation dite involution de Cremona

$$
\sigma: \mathbb{P}^{2}(\mathbb{C})-\rightarrow \mathbb{P}^{2}(\mathbb{C}), \quad(x: y: z) \mapsto(y z: x z: x y)
$$

est birationnelle de degré 2 . On vérifie que

$$
\begin{aligned}
\operatorname{Ind}(\sigma)= & \{(1: 0: 0),(0: 1: 0),(0: 0: 1)\}, \\
\operatorname{Exc}(\sigma)= & \{x=0\} \cup\{y=0\} \cup\{z=0\}, \\
\operatorname{Fix}(\sigma)= & \{(1: 1: 1),(1: 1:-1),(1:-1: 1), \\
& (-1: 1: 1)\} .
\end{aligned}
$$

Le groupe de Jonquières dJ est le sous-groupe maximal de $\operatorname{Bir}\left(\mathbb{P}^{2}(\mathbb{C})\right)$ formé des transformations préservant la fibration $y=$ cte, i.e.

$$
\begin{aligned}
\mathrm{d} \mathrm{J} & \simeq \mathrm{PGL}_{2}(\mathbb{C}(y)) \rtimes \mathrm{PGL}_{2}(\mathbb{C}) \\
= & \left\{\left(\frac{a(y) x+b(y)}{c(y) x+d(y)}, \frac{\alpha y+\beta}{\gamma y+\delta}\right) \mid\right. \\
& {\left.\left[\begin{array}{ll}
a & b \\
c & d
\end{array}\right] \in \mathrm{PGL}_{2}(\mathbb{C}(y)),\left[\begin{array}{ll}
\alpha & \beta \\
\gamma & \delta
\end{array}\right] \in \mathrm{PGL}_{2}(\mathbb{C})\right\} . }
\end{aligned}
$$

On appelle transformation de Jonquières toute transformation birationnelle préservant une fibration rationnelle, ceci étant justifié par le fait que toute fibration rationnelle est birationnellement conjuguée à la fibration $y=$ cte. Lorsque la matrice $\left[\begin{array}{ll}\alpha & \beta \\ \gamma & \delta\end{array}\right]$ représente l'identité, on dit que la transformation correspondante respecte la fibration fibre à fibre. 


\subsection{Feuilletages}

Un feuilletage holomorphe $\mathcal{F}$ de codimension 1 et de degré $\nu$ sur $\mathbb{P}^{2}(\mathbb{C})$ est défini par une 1-forme du type

$$
\omega=u(x, y, z) \mathrm{d} x+v(x, y, z) \mathrm{d} y+w(x, y, z) \mathrm{d} z,
$$

où $u, v$ et $w$ sont des polynômes homogènes de degré $\nu+1$ sans composante commune vérifiant l'identité d'Euler :

$$
x u(x, y, z)+y v(x, y, z)+z w(x, y, z)=0 .
$$

Le lieu singulier $\operatorname{Sing}(\mathcal{F})$ de $\mathcal{F}$ est le projectivisé du lieu singulier de $\omega$

$$
\begin{aligned}
\operatorname{Sing}(\omega)=\left\{(x, y, z) \in \mathbb{C}^{3} \mid\right. \\
u(x, y, z)=v(x, y, z)=w(x, y, z)=0\} .
\end{aligned}
$$

En restriction à la carte affine $z=1$ la 1 -forme $\omega$ s'écrit

$$
u(x, y, 1) \mathrm{d} x+v(x, y, 1) \mathrm{d} y=\widetilde{u} \mathrm{~d} x+\widetilde{v} \mathrm{~d} y+\phi(x \mathrm{~d} y-y \mathrm{~d} x)
$$

où $\widetilde{u}$ et $\widetilde{v}$ sont des polynômes de degré au plus $\nu$ et $\phi$ un polynôme homogène de degré $\nu$.

Soient $\mathcal{F}$ un feuilletage de degré $\nu$ sur le plan projectif complexe, $\mathcal{D}$ une droite générale et $p$ un point de $\mathcal{D}$ non singulier pour $\mathcal{F}$. On dit que $\mathcal{F}$ est transverse à $\mathcal{D}$ en $p$ si la feuille $\mathcal{L}_{p}$ de $\mathcal{F}$ en $p$ est transverse à $\mathcal{D}$ en $p$; sinon on dit que $p$ est un point de tangence entre $\mathcal{D}$ et $\mathcal{F}$. Le degré $\nu$ de $\mathcal{F}$ est exactement le nombre de points de tangence entre $\mathcal{D}$ et $\mathcal{F}$.

La classification des feuilletages de degré 0 ou 1 sur $\mathbb{P}^{2}(\mathbb{C})$ est connue depuis le XIX ${ }^{\text {ème }}$ siècle [Jouanolou 79]. Un feuilletage de degré 0 sur $\mathbb{P}^{2}(\mathbb{C})$ est un pinceau de droites. Tout feuilletage de degré 1 sur le plan projectif complexe possède trois singularités comptées avec multiplicité, a, au moins, une droite invariante et est donné par une 1-forme fermée rationnelle (dit autrement il existe un polynôme homogène $P$ tel que $\omega / P$ soit fermée); les feuilles sont les composantes connexes des «niveaux» d'une primitive de cette 1 -forme. Pour $\nu \geq 2$ peu de propriétés ont été établies, si ce n'est la non existence générique de courbe invariante [Jouanolou 79, Cerveau and Lins Neto 91].

Un point régulier $m$ de $\mathcal{F}$ est dit d'inflexion (pour $\mathcal{F})$ si $\mathcal{L}_{m}$ a un point d'inflexion en $m$; on désigne par Flex $(\mathcal{F})$ l'adhérence de ces points. Présentons un moyen de déterminer cet ensemble Flex $(\mathcal{F})$ donné dans [Pereira 01]. Soit $\mathrm{Z}=E \frac{\partial}{\partial x}+F \frac{\partial}{\partial y}+G \frac{\partial}{\partial z}$ un champ de vecteurs homogène sur $\mathbb{C}^{3}$ non colinéaire au champ radial $\mathrm{R}=x \frac{\partial}{\partial x}+y \frac{\partial}{\partial y}+z \frac{\partial}{\partial z}$ décrivant $\mathcal{F}$, i.e. tel que

$$
\omega=i_{\mathrm{R}} i_{\mathrm{Z}} \mathrm{d} x \wedge \mathrm{d} y \wedge \mathrm{d} z
$$

Définissons le polynôme $\mathcal{H}$ par

$$
\mathcal{H}(x, y, z)=\operatorname{det}\left[\begin{array}{lll}
x & E & \mathrm{Z}(E) \\
y & F & \mathrm{Z}(F) \\
z & G & \mathrm{Z}(G)
\end{array}\right] ;
$$

notons que $\mathcal{H}$ ne dépend pas des choix de $\omega$ et du champ de vecteurs $\mathrm{Z}$ satisfaisant $(\diamond)$, tout du moins à constante multiplicative près. D'après [Pereira 01] le lieu des zéros de $\mathcal{H}$ est constitué de Flex $(\mathcal{F})$ et de l'ensemble des droites invariantes par $\mathcal{F}$.

Soit $\mathcal{F}$ un feuilletage de degré $\nu$ sur $\mathbb{P}^{2}(\mathbb{C})$. L'application de Gauss est l'application rationnelle $\mathcal{G}$ de $\mathbb{P}^{2}(\mathbb{C})$ dans $\breve{\mathbb{P}}^{2}(\mathbb{C})$ qui à un point régulier $m$ associe la tangente $\mathrm{T}_{m} \mathcal{L}_{m}$ à $\mathcal{L}_{m}$ en $m$. Le lieu exceptionnel de $\mathcal{G}$ coïncide avec les droites invariantes par $\mathcal{F}$ et les points d'indétermination de $\mathcal{G}$ sont les points singuliers de $\mathcal{F}$. Un point $m$ est dit générique pour $\mathcal{F}$ s'il est régulier et si $\mathrm{T}_{m} \mathcal{L}_{m}$ a exactement $\nu$ contacts avec $\mathcal{F}$. L'ensemble des points génériques pour $\mathcal{F}$ est le complément de $(\mathcal{H}=0)$. Notons $\Lambda$ le lieu des zéros de $\mathcal{H}$ et $\Lambda^{\prime}$ son image par $\mathcal{G}$. Soit $\mathcal{D}$ un point de $\check{\mathbb{P}}^{2}(\mathbb{C}) \backslash \Lambda^{\prime}$; la fibre $\mathcal{G}^{-1}(\{\mathcal{D}\})$ contient exactement $\nu$ points et la restriction

$$
\mathcal{G}_{\mid \mathbb{P}^{2}(\mathbb{C}) \backslash \Lambda}: \mathbb{P}^{2}(\mathbb{C}) \backslash \Lambda \rightarrow \check{\mathbb{P}}^{2}(\mathbb{C}) \backslash \Lambda^{\prime}
$$

de $\mathcal{G}$ à $\mathbb{P}^{2}(\mathbb{C}) \backslash \Lambda$ est un revêtement. On peut se demander à quelles conditions un tel revêtement possède des automorphismes qui sont birationnels; on donne dans la suite quelques éléments de réponse à cette question.

\section{FEUILLETAGES QUADRATIQUES ET INVOLUTIONS BIRATIONNELLES}

\subsection{Construction de l'Involution et Premières Propriétés}

À tout feuilletage $\mathcal{F}$ de degré 2 défini sur le plan projectif complexe on peut associer une involution birationnelle $\mathcal{I}_{\mathcal{F}}$. En effet considérons un point générique $m$ pour $\mathcal{F}$; le feuilletage étant quadratique $\mathrm{T}_{m} \mathcal{L}_{m}$ est tangente à $\mathcal{F}$ en un second point $p$, l'involution $\mathcal{I}_{\mathcal{F}}$ est la transformation qui échange ces deux points. Plus précisément supposons que $\mathcal{F}$ soit décrit par le champ de vecteurs X. L'image par $\mathcal{I}_{\mathcal{F}}$ d'un point générique pour $\mathcal{F}$ est le point $m+s \mathrm{X}(m)$ où $s$ est l'unique paramètre non nul pour lequel $\mathrm{X}(m)$ et $\mathrm{X}(m+s \mathrm{X}(m))$ sont colinéaires.

Soient $q$ un point singulier de $\mathcal{F}$ et $\mathcal{P}(q)$ le pinceau de droites passant par $q$. La courbe des points de tangence $\operatorname{Tang}(\mathcal{F}, \mathcal{P}(q))$ entre les feuilletages $\mathcal{F}$ et $\mathcal{P}(q)$ est contractée par $\mathcal{I}_{\mathcal{F}}$ sur $q$. On constate que toutes les courbes contractées sont de ce type. Par ailleurs notons $\operatorname{Inv}(\mathcal{F})$ l'ensemble des droites invariantes par $\mathcal{F}$. 
On a l'inclusion $\operatorname{Flex}(\mathcal{F}) \subset \operatorname{Fix}\left(\mathcal{I}_{\mathcal{F}}\right)$. On verra plus loin des exemples où certaines droites de $\operatorname{Inv}(\mathcal{F})$ sont dans $\operatorname{Fix}\left(\mathcal{I}_{\mathcal{F}}\right)$ et d'autres sont contractées par $\mathcal{I}_{\mathcal{F}}$.

Pour $\mathcal{F}$ générique $\mathcal{I}_{\mathcal{F}}$ a sept points d'indétermination correspondant aux sept points singuliers du feuilletage et sept courbes contractées qui sont des cubiques à point double; on reviendra plus loin sur ce point.

Définition 3.1. Soit $\mathcal{F}$ un feuilletage sur $\mathbb{P}^{2}(\mathbb{C})$. Le sousgroupe de $\operatorname{Aut}\left(\mathbb{P}^{2}(\mathbb{C})\right.$ ) qui préserve $\mathcal{F}$ s'appelle le groupe d'isotropie de $\mathcal{F}$; c'est un sous-groupe algébrique de $\operatorname{Aut}\left(\mathbb{P}^{2}(\mathbb{C})\right)$. On le note $\operatorname{Iso}(\mathcal{F})$

$$
\operatorname{Iso}(\mathcal{F})=\left\{\varphi \in \operatorname{Aut}\left(\mathbb{P}^{2}(\mathbb{C})\right) \mid \varphi^{*} \mathcal{F}=\mathcal{F}\right\} .
$$

Remarque 3.2. Soient $\mathcal{F}$ un feuilletage quadratique et $\mathcal{I}_{\mathcal{F}}$ l'involution associée; $\mathcal{I}_{\mathcal{F}}$ commute à tous les éléments de $\operatorname{Iso}(\mathcal{F})$.

\subsection{Classification des Involutions Birationnelles du Plan Projectif Complexe}

Avant d'énoncer le résultat de Bertini repris par Bayle et Beauville, introduisons trois types d'involutions qui, comme on le verra, jouent un rôle très particulier.

Soient $p_{1}, \ldots, p_{7}$ sept points de $\mathbb{P}^{2}(\mathbb{C})$ en position générale, c'est-à-dire satisfaisant les conditions suivantes : il n'y en a pas trois alignés et il n'y en a pas six sur une conique. Désignons par $L$ le système linéaire de cubiques passant par les $p_{i}$; il est de dimension 2 . Soit $p$ un point générique de $\mathbb{P}^{2}(\mathbb{C})$; considérons le pinceau $L_{p}$ constitué des éléments de $L$ passant par $p$. Un pinceau de cubiques génériques ayant neuf points base, on définit par $\mathcal{I}_{G}(p)$ le neuvième point base de $L_{p}$. L'involution $\mathcal{I}_{G}=\mathcal{I}_{G}\left(p_{1}, \ldots, p_{7}\right)$ qui à $p$ associe $\mathcal{I}_{G}(p)$ ainsi construite est appelée involution de Geiser. On peut vérifier qu'une telle involution est birationnelle de degré 8 et que ses points fixes forment une courbe non hyperelliptique de genre 3, de degré 6 avec 7 points doubles ordinaires qui se trouvent en les $p_{i}$. Le lieu exceptionnel d'une involution de Geiser est constitué de sept cubiques passant par les sept points d'indétermination de $\mathcal{I}_{G}$ et singulière en l'un des sept points (cubiques à point double).

Considérons l'ensemble $\mathcal{S}$ des sextiques ayant huit points doubles $p_{1}, \ldots, p_{8}$ en position générale. Fixons un point $m$ de $\mathbb{P}^{2}(\mathbb{C}) ;$ le pinceau des éléments de $\mathcal{S}$ ayant un point double en $m$ contient un dixième point double $m^{\prime}$. L'involution qui échange les points $m$ et $m^{\prime}$ est une involution de Bertini que l'on note $\mathcal{I}_{B}=\mathcal{I}_{B}\left(p_{1}, \ldots, p_{8}\right)$.
Les points fixes de $\mathcal{I}_{B}$ forment une courbe non hyperelliptique de genre 4, de degré 9 avec des points triples en les $p_{i}$, dont la normalisée est isomorphe à une intersection non singulière d'une surface cubique et d'un cône quadratique dans $\mathbb{P}^{3}(\mathbb{C})$.

Pour finir introduisons les involutions de Jonquières. Soit $\mathcal{C}$ une courbe irréductible de degré $\nu \geq 3$. On suppose que $\mathcal{C}$ possède un unique point singulier $p$ qui soit de plus un point multiple ordinaire de multiplicité $\nu-2$. Au couple $(\mathcal{C}, p)$ on va associer une involution birationnelle $\mathcal{I}_{\mathrm{dJ}}$ qui fixe la courbe $\mathcal{C}$ et préserve les droites passant par $p$. Soit $m$ un point générique de $\mathbb{P}^{2}(\mathbb{C})$; notons $q_{m}, r_{m}$ les deux points d'intersection, distincts de $p$, de la droite $(\mathrm{pm})$ avec $\mathcal{C}$. La transformation $\mathcal{I}_{\mathrm{dJ}}$ associe au point $m$ le conjugué harmonique de $m$ sur la droite $(\mathrm{pm})$ par rapport à $q_{m}$ et $r_{m}$; dit autrement le point $\mathcal{I}_{\mathrm{dJ}}(m)$ vérifie la propriété suivante : le birapport de $m, \mathcal{I}_{\mathrm{dJ}}(m), q_{m}$ et $r_{m}$ vaut -1 . La transformation $\mathcal{I}_{\mathrm{dJ}}$ est une involution de Jonquières de degré $\nu$ centrée en $p$ et préservant $\mathcal{C}$. L'ensemble des points fixes de $\mathcal{I}_{\mathrm{dJ}}$ est précisément la courbe $\mathcal{C}$ qui est de genre $\nu-2$ pour $\nu \geq 3$. Pour $\nu=2$ la courbe $\mathcal{C}$ est une conique lisse; on fait alors la même construction en choisissant un point $p$ extérieur à $\mathcal{C}$.

Définition 3.3. On dira qu'une involution est de type projectif si elle est birationnellement conjuguée à une involution projective, de type Jonquières si elle est birationnellement conjuguée à une involution de Jonquières; de même on parlera d'involution de type Bertini, resp. de type Geiser.

L'énoncé suivant donne la classification des involutions birationnelles.

Théorème 3.4. [Bertini 77, Bayle and Beauville 00] Une involution birationnelle est de l'un des quatre types : projectif, Jonquières, Bertini ou Geiser.

Dans [Bayle and Beauville 00] les auteurs montrent aussi que les classes de conjugaison des involutions de $\operatorname{Bir}\left(\mathbb{P}^{2}(\mathbb{C})\right)$ sont uniquement déterminées par le type birationnel des courbes de leurs points fixes.

\subsection{Feuilletages Quadratiques Génériques de $\mathbb{P}^{2}(\mathbb{C})$}

Rappelons qu'un feuilletage quadratique a sept points singuliers comptés avec multiplicité; de plus si on se donne sept points en position générale (dans le même sens que précédemment) il existe un et un seul feuilletage quadratique ayant ces sept points pour points singuliers [Gómez-Mont and Kempf 89]. 
Théorème 3.5. Soient $p_{1}, \ldots, p_{7}$ sept points de $\mathbb{P}^{2}(\mathbb{C})$ en position générale. Soient $\mathcal{F}$ le feuilletage quadratique de $\mathbb{P}^{2}(\mathbb{C})$ dont le lieu singulier est constitué des $p_{i}$ et $\mathcal{I}_{G}$ l'involution de Geiser associée aux $p_{i}$. L'involution $\mathcal{I}_{\mathcal{F}}$ associée à $\mathcal{F}$ et $\mathcal{I}_{G}$ coïncident.

Corollaire 3.6. L'involution associée à un feuilletage quadratique générique sur $\mathbb{P}^{2}(\mathbb{C})$ est une involution de Geiser.

Démonstration du Théorème 3.5. Soit $\Omega$ une 1-forme définissant $\mathcal{F}$

$$
\Omega=u \mathrm{~d} x+v \mathrm{~d} y+w \mathrm{~d} z
$$

avec $u, v, w$ des polynômes homogènes de degrés 3 en $x$, $y$ et $z$ satisfaisant l'identité d'Euler, i.e. $x u+y v+z w=0$.

L'ensemble des cubiques passant par les sept points singuliers est d'après l'hypothèse de généricité un plan projectif. Il s'identifie au projectivisé $\mathbb{P}(\mathrm{E})$ de l'espace vectoriel $\mathrm{E}=\left\{\lambda_{1} u+\lambda_{2} v+\lambda_{3} w \mid \lambda_{i} \in \mathbb{C}\right\}$.

Reprenons l'involution de Geiser $\mathcal{I}_{G}$ associée au 7uplet de points $P=\left(p_{1}, \ldots, p_{7}\right)$. Si $m$ est un point générique du plan projectif complexe, l'ensemble des éléments de $\mathbb{P}(\mathrm{E})$ passant par $m$ est un pinceau $\mathbb{P}(\mathrm{E}(m))$ avec

$$
\begin{aligned}
\mathrm{E}(m)=\left\{\lambda_{1} u+\lambda_{2} v+\lambda_{3} w \mid \lambda_{i} \in \mathbb{C},\right. \\
\left.\lambda_{1} u(\widetilde{m})+\lambda_{2} v(\widetilde{m})+\lambda_{3} w(\widetilde{m})=0\right\}
\end{aligned}
$$

où $\widetilde{m}$ est un relevé de $m$ à $\mathbb{C}^{3} \backslash\{0\}$. Ce pinceau contient les $p_{i}, m$ et donc un autre point base noté $m^{\prime}$. L'involution de Geiser est la transformation qui à $m$ associe $m^{\prime}$. Remarquons que $\mathrm{E}(m)=\mathrm{E}\left(m^{\prime}\right)$ et que par suite $\Omega(\widetilde{m})$ et $\Omega\left(\widetilde{m}^{\prime}\right)$ sont $\mathbb{C}$-colinéaires, i.e. les plans $\operatorname{ker} \Omega(\widetilde{m})$ et ker $\Omega\left(\widetilde{m}^{\prime}\right)$ sont identiques. Notons aussi que l'identité d'Euler assure que $\widetilde{m}$ et $\widetilde{m}^{\prime}$ appartiennent à ce plan. Il en résulte que $\operatorname{ker} \Omega(\widetilde{m})=\operatorname{ker} \Omega\left(\widetilde{m}^{\prime}\right)$ est le plan défini par $\widetilde{m}$ et $\widetilde{m}^{\prime}$. Ceci se traduit sur $\mathbb{P}^{2}(\mathbb{C})$ de la façon suivante : la droite $\left(m m^{\prime}\right)$ dans $\mathbb{P}^{2}(\mathbb{C})$ est tangente à $\mathcal{F}$ en $m$ et $m^{\prime}$; par conséquent l'involution de Geiser $\mathcal{I}_{G}$ est exactement l'involution $\mathcal{I}_{\mathcal{F}}$ associée au feuilletage $\mathcal{F}$.

Les deux remarques qui suivent précisent ce qui est annoncé au §3.1.

Remarque 3.7. Si $\mathcal{F}$ est un feuilletage quadratique, le polynôme $\mathcal{H}$ associé à $\mathcal{F}$ est de degré 6 ; génériquement un tel $\mathcal{F}$ n'a pas de droite invariante [Jouanolou 79 , Cerveau and Lins Neto 91] donc $\operatorname{Flex}(\mathcal{F})$ et $(\mathcal{H}=0)$ coïncident. D'autre part comme $\mathcal{I}_{\mathcal{F}}$ est de type Geiser sa courbe de points fixes est une courbe irréductible de degré 6 , ce qui force l'inclusion $\operatorname{Flex}(\mathcal{F}) \subset \operatorname{Fix}\left(\mathcal{I}_{\mathcal{F}}\right)$ à être une égalité.
Remarque 3.8. Supposons que l'origine de la carte $z=1$ soit un point singulier de $\mathcal{F}$. Comme $\mathcal{F}$ est générique, il est, pour un bon choix de coordonnées affines, décrit par

$$
\mathrm{X}=(x+\cdots) \frac{\partial}{\partial x}+(a y+\cdots) \frac{\partial}{\partial y}, \quad a \notin\{0,1\} .
$$

Soit $t \mapsto(x(t), y(t))$ une courbe intégrale locale de $\mathrm{X}$ qui n'est pas une droite; les points d'inflexion sur cette courbe intégrale sont donnés par $\dot{x} \ddot{y}-\dot{y} \ddot{x}=0$. L'ensemble Flex $(\mathcal{F})$ est donc décrit au voisinage de l'origine par $a(a-$ 1) $x y+\cdots=0$. Il en résulte que génériquement $\operatorname{Flex}(\mathcal{F})$, et donc $\operatorname{Fix}\left(\mathcal{I}_{\mathcal{F}}\right)$, est une sextique avec 7 points nodaux ce qui est un résultat classique.

\subsection{Exemples Explicites d'Involution de Geiser}

Soit $\mathcal{F}$ un feuilletage quadratique générique sur $\mathbb{P}^{2}(\mathbb{C}) ;$ on peut supposer à conjugaison linéaire près que $(1: 0$ : $0),(0: 1: 0),(0: 0: 1)$ et $(1: 1: 1)$ sont singuliers pour $\mathcal{F}$. Le feuilletage $\mathcal{F}$ est alors défini par

$$
\begin{aligned}
& \left(\varepsilon x^{2} y+a x^{2}+b x y+c x+e y\right) \frac{\partial}{\partial x} \\
& \quad+\left(\varepsilon x y^{2}+A y^{2}+B x y+C x+E y\right) \frac{\partial}{\partial y} .
\end{aligned}
$$

Puisque génériquement la droite à l'infini n'est pas préservée par $\mathcal{F}$ le coefficient $\varepsilon$ est non nul et on se ramène à $\varepsilon=1$; de plus on a $1+a+b+c+e=1+A+B+C+E=0$, ces deux dernières conditions assurant que $(1: 1: 1)$ est singulier.

L'involution associée $\mathcal{I}_{\mathcal{F}}$ s'écrit $\left(\frac{U_{1}}{T U_{2}}, \frac{V_{1}}{T V_{2}}\right)$ avec

$$
\begin{aligned}
U_{1}= & (B-a)(E-A(B-a)) x^{3} y^{2} \\
+ & \left(E\left(a B-a^{2}+C\right)+2 A C(a-B)\right) x^{3} y \\
+ & C(a E-A C) x^{3}+e(A E-b E-c A+e B) y^{3} \\
+ & \left(2\left(A^{2} c-e E-b c A-A^{2} E\right)-a e A+c e+b e B\right. \\
& \left.+3 b A E-b^{2} E\right) x y^{3}+(c E-e C)(E-c) x y \\
+ & e(e C-c E) y^{2}+\left(a e B-e C-e B^{2}+E^{2}-c E+b B E\right. \\
& +2\left(b A C-a c A-A^{2} C-a b E-A B E+c A B\right) \\
& +3 a A E) x^{2} y^{2}+C(c E-e C) x^{2} \\
+ & \left(2(c A C-e C B-a c E-A C E)+a E^{2}+c B E+b C E\right. \\
& +a e C) x^{2} y+(2 A(A-b)(a-B)+e(a-B) \\
& +(A-b) E) x^{2} y^{3}+(b-A)(e-A(b-A)) x y^{4} \\
+ & \left(b e C-a e E-c^{2} A+b E^{2}-A E^{2}+3 c A E+c e B-e B E\right. \\
& -2 b c E) x y^{2}+e\left(A^{2}-b A+e\right) y^{4},
\end{aligned}
$$




$$
\begin{aligned}
V_{1}= & (2 a(a-B)(b-A)-a c-A C+b C+c B) x^{3} y^{2} \\
+ & (A-b)(a(A-b)+c) x^{2} y^{3} \\
+ & (a-B)(C+a(a-B)) x^{4} y \\
+ & \left(2\left(a B E+c C-a^{2} E+a^{2} c\right)-3 a c B-C E+a A C\right. \\
& \left.-b B C+c B^{2}\right) x^{3} y+C(c E-e C) x^{2} \\
+ & (E-c)(c E-e C) x y+(2(a A E-a e B+a b c \\
& \left.-a b E+c A B+a^{2} e\right)+b^{2} C+e C-b c B-c^{2}-3 a c A \\
& -b A C+c E) x^{2} y^{2}-C\left(C-a B+a^{2}\right) x^{4} \\
+ & \left(2 c B E+a E^{2}-b C E-3 a c E-c^{2} B+b c C+c A C\right. \\
& \left.-e C B+a c^{2}\right) x^{2} y+C(a E-b C-a c+c B) x^{3} \\
+ & e(a e-c A) y^{3}+\left(2(a c e+b e C-a e E+c A E)-c^{2} A\right. \\
& -c e B-e A C-b c E) x y^{2} \\
+ & \left(c A^{2}-c e-b c A+2 a e(b-A)\right) x y^{3}+e(e C-c E) y^{2}, \\
& \quad \\
& \quad U_{2}=x y^{2}+A y^{2}+B x y+C x+E y, \\
& \quad V_{2}=x^{2} y+a x^{2}+b x y+c x+e y, \\
& \\
& +\left(C-a B+a^{2}\right) x^{2}+(E-c-A B+a b) x y \\
& +(b C-c B-A C+a c) x+\left(b A-A^{2}-e\right) y^{2} \\
& +(b E-e B-A E+a e) y .
\end{aligned}
$$

Pour des choix génériques des coefficients ceci permet d'avoir des exemples explicites d'involutions de Geiser «normalisées » au sens où 4 des points d'indétermination parmi les 7 sont fixés comme précédemment. Voici comment on procède. On choisit dans $\mathbb{C}^{2} \subset \mathbb{P}^{2}(\mathbb{C})$ trois nouveaux points $m_{1}, m_{2}, m_{3}$ de sorte que dans nos sept points il n'y ait pas de configurations de 3 points en alignement. On cherche alors des coefficients $a, b, c, A, B$, $C$ tels que le champ X correspondant s'annule en les $m_{i}$. Si $m_{i}=\left(x_{i}, y_{i}\right)$ on doit résoudre le système linéaire

$$
\left\{\begin{array}{l}
\left(x_{i}^{2}-y_{i}\right) a+\left(x_{i} y_{i}-y_{i}\right) b+\left(x_{i}-y_{i}\right) c=y_{i}-x_{i}^{2} y_{i}, \\
\left(y_{i}^{2}-y_{i}\right) A+\left(x_{i} y_{i}-y_{i}\right) B+\left(x_{i}-y_{i}\right) C=y_{i}-x_{i} y_{i}^{2},
\end{array}\right.
$$

avec $i=1,2,3$. Notons $D\left(m_{1}, m_{2}, m_{3}\right)$ le déterminant de la matrice associée. Si les points $m_{i}$ sont choisis de sorte que $D\left(m_{1}, m_{2}, m_{3}\right)$ soit non nul, on peut trouver le champ $\mathrm{X}$ recherché. Il reste ensuite à vérifier que $\mathrm{X}$ définit bien un feuilletage quadratique c'est-à-dire que les composantes de $\mathrm{X}$ sont sans facteur commun. On est alors assuré que pour ces 7 points, il n'y a pas de conique passant par 6 d'entre eux; en effet si $\mathcal{C}$ est une conique lisse et $\mathcal{F}$ un feuilletage quadratique sur le plan projectif complexe, $\mathcal{C}$ contient au plus cinq points singuliers de $\mathcal{F}$ (voir [Campillo and Olivares 00]). On constate que ces involutions sont bien de degré 8 .

Avec les notations précédentes l'énoncé qui suit résume la procédure qui n'utilise que de l'algèbre linéaire.

Proposition 3.9. Soient $m_{1}, m_{2}, m_{3}$ trois points génériques dans $\mathbb{C}^{2}$. Soit $(a, b, c, A, B, C)$ une solution de (3-1). Alors l'involution associée aux sept points (1:0: $0),(0: 1: 0),(0: 0: 1),(1: 1: 1), m_{1}, m_{2}$ et $m_{3}$ est donnée par $\left(\frac{U_{1}}{T U_{2}}, \frac{V_{1}}{T V_{2}}\right)$. C'est une involution de Geiser.

Remarque 3.10. Dans [Williams 20] l'auteur explique comment, lorsque les $m_{i}$ sont en position spéciale, l'involution dégénère (baisse du degré, courbes invariantes,...).

Exemple 3.11. $\mathrm{Si} m_{1}=\left(\frac{1}{2}, \frac{3}{4}\right), m_{2}=\left(\frac{3}{4}, \frac{1}{2}\right)$ et $m_{3}=$ $\left(\frac{4}{3}, \frac{2}{3}\right)$, l'involution associée $\mathcal{I}_{\mathcal{F}}$ s'écrit alors $\left(\frac{U_{1}}{T U_{2}}, \frac{V_{1}}{T V_{2}}\right)$ avec

$$
\begin{aligned}
U_{1}= & 1220 x^{3} y^{2}-1844 x^{3} y+693 x^{3}-2456 x^{2} y^{3}+3624 x^{2} y^{2} \\
& -1054 x^{2} y-198 x^{2}+1184 x y^{4}-1768 x y^{3}+259 x y^{2} \\
& +286 x y+144 y^{4}-36 y^{2}-54 y^{3}, \\
V_{1}= & 976 x^{4} y-792 x^{4}+1988 x^{3} y^{2}-5456 x^{3} y+3267 x^{3} \\
& -3848 x^{2} y^{3}+5652 x^{2} y^{2}+242 x^{2} y-2178 x^{2}+2936 x y^{3} \\
& -5951 x y^{2}+3146 x y+414 y^{3}-396 y^{2}, \\
& U_{2}=x y^{2}-\frac{47}{18} y^{2}+\frac{13}{18} x y-x+\frac{17}{9} y, \\
& V_{2}=-\frac{101}{18} x^{2}+11 x^{2} y-\frac{221}{18} x y+\frac{44}{9} x+2 y, \\
& T=-2\left(36 x^{2}-378 x y+198 x+396 y^{2}-252 y\right) ;
\end{aligned}
$$

c'est une involution de Geiser.

Dans les paragraphes qui suivent, on s'intéresse aux involutions associées à des feuilletages ayant des propriétés, par exemple de symétries, spéciales.

\subsection{Feuilletage Quadratique de Jouanolou}

Le feuilletage $\mathcal{F}_{J}$ est décrit dans la carte $z=1$ par la 1 -forme

$$
\omega_{J}=\left(x^{3}-y^{2}\right) \frac{\partial}{\partial x}+\left(x^{2} y-1\right) \frac{\partial}{\partial y} ;
$$

cet exemple est dû à Jouanolou [Jouanolou 79]. Historiquement c'est le premier exemple connu de feuilletage 
sans courbe algébrique invariante [Jouanolou 79]; c'est aussi un feuilletage qui n'admet pas d'ensemble minimal non trivial si l'on en croit [Camacho and de Figueiredo 01].

L'involution associée $\mathcal{I}_{\mathcal{F}_{J}}$ est donnée par

$$
\begin{gathered}
\left(x y^{7}+3 x^{5} y^{2} z-x^{8}-5 x^{2} y^{4} z^{2}+2 y^{3} z^{5}+x^{3} y z^{4}-x z^{7}:\right. \\
3 x y^{5} z^{2}+2 x^{5} z^{3}-x^{7} y-5 x^{2} y^{2} z^{4}+x^{4} y^{3} z+y z^{7}-y^{8}: \\
\left.x y^{4} z^{3}-5 x^{4} y^{2} z^{2}-y^{7} z+2 x^{3} y^{5}+3 x^{2} y z^{5}-z^{8}+x^{7} z\right) .
\end{gathered}
$$

Elle est de degré 8; par ailleurs $\operatorname{Ind}\left(\mathcal{I}_{\mathcal{F}_{J}}\right)=\operatorname{Sing}\left(\mathcal{F}_{J}\right)=$ $\left\{\left(\xi^{j}: \xi^{-2 j}: 1\right) \mid j=0, \ldots, 6\right\}$ où $\xi$ désigne une racine 7 ième de l'unité.

On constate que $\mathcal{H}(x, y, z)=2\left(3 x^{2} y^{2} z^{2}-x y^{5}-x^{5} z-\right.$ $\left.y z^{5}\right)$; puisque $\mathcal{F}_{J}$ n'a pas de courbe algébrique invariante Flex $\left(\mathcal{F}_{J}\right)$ coïncide avec le lieu des zéros de $\mathcal{H}$ et c'est aussi $\operatorname{Fix}\left(\mathcal{I}_{\mathcal{F}_{J}}\right)$. Le point $(1: 1: 1)$ est un point singulier de Flex $\left(\mathcal{F}_{J}\right)$ de type point double ordinaire. En faisant agir le groupe d'isotropie de $\mathcal{F}_{J}$

$$
\operatorname{Iso}\left(\mathcal{F}_{J}\right)=\left\langle(y: z: x),\left(\xi^{j} x: \xi^{-2 j} y: z\right) \mid j=0, \ldots, 6\right\rangle
$$

on constate que chaque point singulier de $\mathcal{F}_{J}$ est un point double ordinaire de $\operatorname{Flex}\left(\mathcal{F}_{J}\right)$; on peut vérifier que ce sont les seuls points singuliers de $\operatorname{Flex}\left(\mathcal{F}_{J}\right)$. Le lecteur se convaincra aisément de l'irréductibilité de $\mathcal{H}$. Il s'en suit que Flex $\left(\mathcal{F}_{J}\right)$ est irréductible et de genre $\frac{(6-1)(6-2)}{2}-7=$ 3. Les points de $\operatorname{Sing}\left(\mathcal{F}_{J}\right)$ sont par l'argument précédent en position générale et par conséquent l'involution $\mathcal{I}_{\mathcal{F}_{J}}$ est une involution de Geiser. Le groupe engendré par $\mathcal{I}_{\mathcal{F}_{J}}$ et $\operatorname{Iso}\left(\mathcal{F}_{J}\right)$ est un groupe fini qui ne peut être conjugué à un sous-groupe $\operatorname{de} \operatorname{Aut}\left(\mathbb{P}^{2}(\mathbb{C})\right)$ par une transformation birationnelle (le genre 3 des points fixes de $\mathcal{I}_{\mathcal{F}_{J}}$ est une obstruction). Ce groupe apparait dans la classification des sous-groupes finis de $\operatorname{Bir}\left(\mathbb{P}^{2}(\mathbb{C})\right.$ ) (voir [Dolgachev and Iskovskikh 10]).

On peut donc énoncer le résultat suivant.

Proposition 3.12. L'involution $\mathcal{I}_{\mathcal{F}_{J}}$ associée au feuilletage de Jouanolou de degré 2 est de type Geiser. Le groupe engendré par $\mathcal{I}_{\mathcal{F}_{J}}$ et le groupe d'isotropie de $\mathcal{F}_{J}$ est un sous-groupe fini d'ordre 42, non linéarisable du groupe de Cremona.

Si l'involution associée à un feuilletage quadratique est précisément de degré 8 , est-elle de type Geiser ? La réponse, négative, est donnée au §3.6.2.

\subsection{Feuilletages Quadratiques de $\mathbb{P}^{2}(\mathbb{C})$ Ayant une Unique Singularité}

Contrairement au paragraphe précédent consacré au cas générique, on s'intéresse ici à une catégorie de feuille- tages quadratiques très particuliers, ceux qui comptent une seule singularité; la classification de ces éléments a été établie dans [Cerveau et al. 09].

Théorème 3.13. [Cerveau et al. 09] $\grave{A}$ automorphisme de $\mathbb{P}^{2}(\mathbb{C})$ près, il y a quatre feuilletages quadratiques sur $\mathbb{P}^{2}(\mathbb{C})$ ayant une seule singularité ; ils sont décrits par les 1-formes suivantes

$$
\begin{aligned}
& \omega_{1}=x^{2} \mathrm{~d} x+y^{2}(x \mathrm{~d} y-y \mathrm{~d} x) \\
& \omega_{2}=x^{2} \mathrm{~d} x+\left(x+y^{2}\right)(x \mathrm{~d} y-y \mathrm{~d} x) \\
& \omega_{3}=x y \mathrm{~d} x+\left(x^{2}+y^{2}\right)(x \mathrm{~d} y-y \mathrm{~d} x) \\
& \omega_{4}=\left(x+y^{2}-x^{2} y\right) \mathrm{d} y+x\left(x+y^{2}\right) \mathrm{d} x .
\end{aligned}
$$

On désigne par $\mathcal{F}_{k}$ le feuilletage associé à $\omega_{k}$. Dans chaque éventualité le point singulier est situé en l'origine $: \operatorname{Sing}\left(\mathcal{F}_{k}\right)=\{(0: 0: 1)\}$. Les trois premiers feuilletages produisent des involutions de Jonquières de degré inférieur ou égal à 4. Examinons par exemple l'involution associée à $\mathcal{F}_{1}$.

3.6.1 Le Feuilletage $\mathcal{F}_{1}$ et Son Involution $\mathcal{I}_{\mathcal{F}_{1}}$. L'involution associée à $\mathcal{F}_{1}$ est $\mathcal{I}_{\mathcal{F}_{1}}=\left(x^{3}:-x^{2} y: x^{2} z-2 y^{3}\right)$; on remarque qu'elle préserve la fibration $y / x=$ cte. Elle est de degré trois et ses points fixes $\operatorname{Fix}\left(\mathcal{I}_{\mathcal{F}_{1}}\right)$ coïncident avec la droite $y=0$; c'est exactement l'ensemble des points d'inflexion de $\mathcal{F}_{1}$. L'unique droite contractée par $\mathcal{I}_{\mathcal{F}_{1}}$ est la droite d'équation $x=0$. La transformation $\mathcal{I}_{\mathcal{F}_{1}}$ s'écrit $\left(-y, z-2 y^{3}\right)$ dans la carte affine $x=1$; elle est conjuguée à $(-y, z)$ via $\ell_{1}=\left(y, z+y^{3}\right)$.

On sait, d'après [Cerveau et al. 09], que $\operatorname{Iso}\left(\mathcal{F}_{1}\right)=$ $\left\{\left(\beta^{3} x: \beta^{2} y: z+\gamma x\right) \mid \gamma \in \mathbb{C}, \beta \in \mathbb{C}^{*}\right\} ;$ ce groupe est isomorphe au groupe des transformations affines de la droite. On constate que tout élément de $\operatorname{Iso}\left(\mathcal{F}_{1}\right)$ est invariant par conjugaison par $\ell_{1}$; autrement dit le groupe $\left\langle\operatorname{Iso}\left(\mathcal{F}_{1}\right), \mathcal{I}_{\mathcal{F}_{1}}\right\rangle$ engendré par $\operatorname{Iso}\left(\mathcal{F}_{1}\right)$ et $\mathcal{I}_{\mathcal{F}_{1}}$ est linéarisable $\left(\right.$ via $\left.\ell_{1}\right)$ dans $\operatorname{Bir}\left(\mathbb{P}^{2}(\mathbb{C})\right)$.

3.6.2 Le feuilletage $\mathcal{F}_{4}$ et I'Involution $\mathcal{I}_{\mathcal{F}_{4}}$. Le feuilletage $\mathcal{F}_{4}$ induit l'involution de degré 8 donnée par

$$
\begin{aligned}
& \mathcal{I}_{\mathcal{F}_{4}}=\left(\left(x z+y^{2}\right)\left(x y z+x^{3}+y^{3}\right)^{2}:\right. \\
& \quad\left(\left(2 x^{2}-y z\right)\left(x y z+x^{3}+y^{3}\right)\right. \\
& \left.\quad-x^{5}+x^{3} y z-x^{2} z^{3}-x y^{2} z^{2}\right)\left(x y z+x^{3}+y^{3}\right) \\
& \quad: x y^{7}-x^{7} y-3 x y^{4} z^{3}-3 x^{2} y^{2} z^{4}+4 x^{4} y z^{3}+6 x^{2} y^{5} z \\
& \left.\quad+9 x^{3} y^{3} z^{2}-x^{4} y^{4}+x^{5} y^{2} z-x^{3} z^{5}+2 x^{6} z^{2}-y^{6} z^{2}\right) .
\end{aligned}
$$

Comme on l'a dit précédemment un feuilletage quadratique générique produit une involution de Geiser de degré 8 donc. Le feuilletage $\mathcal{F}_{4}$ produit une involution de 
degré 8 : ainsi la dégénérescence du feuilletage ne conduit pas toujours à une perte de degré de l'involution associée. Toutefois $\mathcal{I}_{\mathcal{F}_{4}}$ n'est pas de type Geiser. Elle possède un seul point d'indétermination et son ensemble exceptionnel $\operatorname{Exc}\left(\mathcal{I}_{\mathcal{F}_{4}}\right)=\left\{x y z+x^{3}+y^{3}=0\right\}$ est une cubique à point double.

Un calcul montre que $\mathcal{H}(x, y, z)=x^{4} y z+x^{3} y^{3}+x^{6}-$ $3 x y^{4} z-y^{6}-x^{3} z^{3}-3 x^{2} y^{2} z^{2}$. D'après [Cerveau et al. 09] le feuilletage $\mathcal{F}_{4}$ n'admet pas de courbe algébrique invariante, par suite $\operatorname{Flex}\left(\mathcal{F}_{4}\right)=\operatorname{Fix}\left(\mathcal{I}_{\mathcal{F}_{4}}\right)$ et $(\mathcal{H}=0)$ coïncident.

Le changement de variables $\left(y, z-y^{2}\right)$ permet de constater que $\operatorname{Flex}\left(\mathcal{F}_{4}\right)=\operatorname{Fix}\left(\mathcal{I}_{\mathcal{F}_{4}}\right)$ est une courbe rationnelle et $\mathcal{I}_{\mathcal{F}_{4}}$ est de type projectif.

Le groupe d'isotropie de $\mathcal{F}_{4}$ est donné par $\operatorname{Iso}\left(\mathcal{F}_{4}\right)=$ $\left\{\mathrm{id},\left(\mathbf{j} x: \mathbf{j}^{2} y: z\right),\left(\mathbf{j}^{2} x: \mathbf{j} y: z\right)\right\}$, où $\mathbf{j}=\mathrm{e}^{2 \mathbf{i} \pi / 3}$, et commute, comme on l'a dit, à $\mathcal{I}_{\mathcal{F}_{4}}$. Le groupe engendré par $\mathcal{I}_{\mathcal{F}_{4}}$ et $\operatorname{Iso}\left(\mathcal{F}_{4}\right)$ est un sous-groupe abélien à huit éléments de $\operatorname{Bir}\left(\mathbb{P}^{2}(\mathbb{C})\right)$.

\subsection{Feuilletages et Pinceaux de Coniques}

Les feuilletages $\mathcal{F}$ associés à des pinceaux de coniques jouent un rôle particulier dans la classification des feuilletages quadratiques car leur ensemble Flex est vide; les zéros de $\mathcal{H}$ sont donc des droites invariantes (six pour un pinceau générique). L'exemple qui suit montre que ces droites peuvent jouer pour l'involution associée $\mathcal{I}_{\mathcal{F}}$ des rôles différents et aussi qu'à l'inverse du cas générique $\operatorname{Flex}(\mathcal{F})$ et $\operatorname{Fix}\left(\mathcal{I}_{\mathcal{F}}\right)$ peuvent être différents.

On considère le feuilletage $\mathcal{F}_{5}$ défini par le pinceau $\frac{y^{2}-x z}{x y}=$ cte. L'involution $\mathcal{I}_{\mathcal{F}_{5}}$ associée est donnée par $\mathcal{I}_{\mathcal{F}_{5}}=\left(-x^{2}: x y: x z+2 y^{2}\right)$. On constate que la droite $y=0$, qui est invariante par $\mathcal{F}_{5}$, est une droite de points fixes de $\mathcal{I}_{\mathcal{F}_{5}}$. Par contre la droite $x=0$, qui est elle aussi invariante, est contractée par $\mathcal{I}_{\mathcal{F}_{5}}$ sur le point $(0: 0: 1)$.

On remarque que

$$
\begin{aligned}
& \operatorname{Iso}\left(\mathcal{F}_{5}\right) \\
& \quad=\left\{\left(\gamma^{2} x, \gamma y\right),\left(\frac{x}{1+\beta y}, \frac{y}{1+\beta y}\right) \mid \gamma \in \mathbb{C}^{*}, \beta \in \mathbb{C}\right\} .
\end{aligned}
$$

En se plaçant dans la carte $x=1$ on montre que $\mathcal{I}_{\mathcal{F}_{5}}$ est conjuguée à $(x:-y:-z)$ par la transformation $\left(x^{2}\right.$ : $\left.-y x: x z-y^{2}\right)$. Il se trouve que cette application commute à $\operatorname{Iso}\left(\mathcal{F}_{5}\right)$; le groupe engendré par $\operatorname{Iso}\left(\mathcal{F}_{5}\right)$ et $\mathcal{I}_{\mathcal{F}_{5}}$ est ici linéarisable.

\section{FEUILLETAGES DE DEGRÉ SUPÉRIEUR ET INVOLUTIONS}

Soit $\mathcal{I}$ une involution birationnelle que l'on écrit $\mathcal{I}=$ $\left(\mathcal{I}_{1}, \mathcal{I}_{2}\right)$ dans la carte affine $z=1$. On suit une démarche inverse à la précédente : on associe à $\mathcal{I}$ le feuilletage $\mathcal{F}$ défini par le champ de vecteurs rationnel

$$
\left(x-\mathcal{I}_{1}(x, y)\right) \frac{\partial}{\partial x}+\left(y-\mathcal{I}_{2}(x, y)\right) \frac{\partial}{\partial y} .
$$

Soient $\nu$ le degré de $\mathcal{F}$ et $\mathcal{D}$ une droite générique $; \mathcal{F}$ a, par définition du degré, $\nu$ points de tangence avec $\mathcal{D}$. Si $q$ est l'un de ces points, alors par construction son image $\mathcal{I}(q)$ par $\mathcal{I}$ est aussi un point de tangence; par suite $\nu$ est pair.

Les degrés de $\mathcal{I}$ et $\mathcal{F}$ sont reliés comme suit : $\operatorname{deg} \mathcal{F} \leq$ $\operatorname{deg} \mathcal{I}-\operatorname{deg} \operatorname{Fix}(\mathcal{I})$. Si $\operatorname{Fix}(\mathcal{I})$ est irréductible, $\operatorname{deg} \mathcal{I}-$ $\operatorname{deg} \mathcal{F}$ est un multiple de $\operatorname{deg} \operatorname{Fix}(\mathcal{I})$. On en déduit par exemple que le feuilletage associé à une involution de Bertini est de degré $17-9=8$. Bien sûr si $\mathcal{I}$ est une involution de Geiser précisément de degré 8 on retombe sur la construction associée aux feuilletages quadratiques. On observe donc, lorsque le degré de $\mathcal{F}$, noté $2 n$, est strictement plus grand que 2 , le phénomène suivant : chaque droite générique contient $n$ orbites distinctes suivant $\mathcal{I}$. Il en est ainsi pour les involutions de Bertini où les orbites sont arrangées en constellations de 4 orbites en alignement.

Le degré du feuilletage associé à l'involution de Cremona $\sigma=\left(\frac{1}{x}, \frac{1}{y}\right)$ est aussi 2 , ceci étant lié au fait que les points fixes de $\sigma$ sont isolés.

Il ne faut pas croire que le feuilletage associé détermine l'involution comme le montrent les exemples qui suivent; soit $R \in \mathbb{C}(x)$ une fonction rationnelle de degré quelconque. Le feuilletage associé à l'involution $\left(x, \frac{R(x)}{y}\right)$ est le pinceau de droites $x=$ cte et ceci indépendamment du choix de $R$. Toutefois il s'agit de cas exceptionnels.

L'involution de type de Jonquières

$$
\mathcal{I}=\left(\frac{y}{1+x^{2} y^{2}}, x\left(1+x^{2} y^{2}\right)\right)
$$

est de degré 9 . Elle préserve la fibration $x y=$ cte fibre à fibre. Les points fixes de $\mathcal{I}$ sont donnés par $\operatorname{Fix}(\mathcal{I})=$ $\left\{y-x-x^{3} y^{2}=0\right\}$. Le lieu d'indétermination de $\mathcal{I}$ est constitué des points $(1: 0: 0)$ et $(0: 1: 0)$ et son lieu exceptionnel des courbes $z=0$ et $z^{4}+x^{2} y^{2}=0$.

Le feuilletage $\mathcal{F}$ associé à cette involution est de degré 4 et décrit par le champ

$$
-\frac{\partial}{\partial x}+\left(1+x^{2} y^{2}\right) \frac{\partial}{\partial y}
$$

Il définit un feuilletage de Ricatti sur $\mathbb{P}^{1}(\mathbb{C}) \times \mathbb{P}^{1}(\mathbb{C})$ : il est en effet transverse à la fibration $x=$ cte. L'équation différentielle induite, après réduction à une équation 


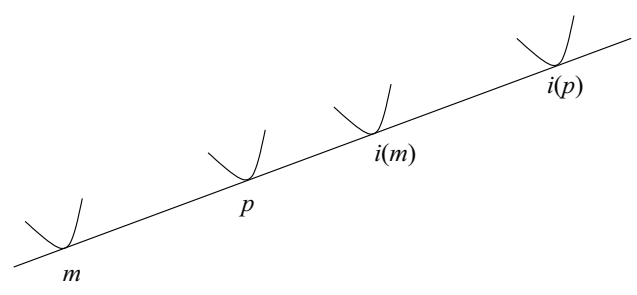

FIGURE 1. Points de contacts.

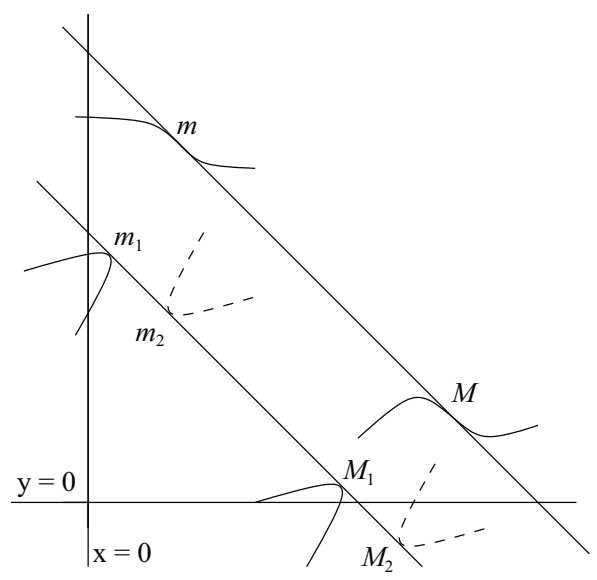

FIGURE 2. Bifurcation des points de contacts.

linéaire du second ordre, s'intègre via les fonctions de Bessel.

Si $\mathcal{D}$ est une droite générale les quatre points de tangences de $\mathcal{D}$ et $\mathcal{F}$ constituent deux orbites de $\mathcal{I}$ (voir figure 1).

Avec les notations habituelles on obtient

$$
\mathcal{H}(x, y, z)=2 x y z^{5}\left(x z^{4}-y z^{4}+x^{3} y^{2}\right) .
$$

Les droites $x=0$ et $y=0$ ne sont pas invariantes par le feuilletage, par contre $z=0$ l'est. Par suite $\operatorname{Flex}(\mathcal{F})$ est l'union des courbes $x=0, y=0$ et $x-y-x^{3} y^{2}=0$; cette dernière courbe, qui est précisément l'ensemble $\operatorname{Fix}(\mathcal{I})$, est elliptique. On constate que les courbes de points d'inflexion $x=0$ et $y=0$ ne font pas partie des points fixes de notre involution $\mathcal{I}$. En voici l'explication : les courbes $x=0$ et $y=0$ sont en fait échangées par $\mathcal{I}$.

On a bifurcation de deux paires de points de contacts simples $m_{1}, m_{2}$ et $M_{1}, M_{2}$ vers les points d'inflexion $m$ et $M$; à l'inverse du cas quadratique, l'involution n'échange pas $m_{1}$ et $m_{2}$ (respectivement $M_{1}$ et $M_{2}$ ), mais disons $m_{1}$ et $M_{1}$ (respectivement $m_{2}$ et $M_{2}$ ), voir figure 2 .

\section{FEUILLETAGES DE DEGRÉ 3 ET TRIVOLUTIONS}

\subsection{Classification des Trivolutions Birationnelles}

Les transformations birationnelles périodiques de période 3, encore appelées trivolutions birationnelles, ont été classées dans [de Fernex 04]: une trivolution birationnelle $\mathcal{T}$ est à conjugaison birationnelle près

- ou bien un automorphisme de $\mathbb{P}^{2}(\mathbb{C})$;

- ou bien une trivolution de Jonquières, i.e. une transformation birationnelle d'ordre 3 qui préserve une fibration rationnelle;

- ou bien un automorphisme sur une surface de del Pezzo de degré 3 définie par une équation de la forme $x^{3}=F(y, z, w)$ dans $\mathbb{P}^{3}(\mathbb{C})$ avec $F$ polynôme homogène de degré 3 à singularité isolée; dans ce cas $\mathcal{T}$ est la restriction de l'automorphisme de $\mathbb{P}^{3}(\mathbb{C})$ défini par $(\xi x: y: z: w)$, avec $\xi^{3}=1$, (la courbe de points fixes de $\mathcal{T}$ est alors isomorphe à la courbe elliptique $\Gamma=\left\{(y: z: w) \in \mathbb{P}^{2}(\mathbb{C}) \mid F(y, z, w)=\right.$ $0\})$;

- ou bien un automorphisme sur une surface de del Pezzo de degré 6 décrite par $z^{3}=w^{2}+F_{6}(x, y, w)$ (avec $F_{6}$ polynôme homogène de degré 6 ) dans l'espace projectif à poids $\mathbb{P}(1,1,2,3)$ muni des coordonnées $(x, y, z, w)$; alors $\mathcal{T}$ est la restriction de l'automorphisme de $\mathbb{P}(1,1,2,3)$ donné par $(x: y:$ $\xi z: w)$, avec $\xi^{3}=1$.

\subsection{Construction de Trivolutions et Premières Propriétés}

Soit $\mathcal{F}$ un feuilletage de degré 3 sur $\mathbb{P}^{2}(\mathbb{C})$; toute droite générique de $\mathbb{P}^{2}(\mathbb{C})$ est tangente à $\mathcal{F}$ en trois points. L"'application" qui échange ces trois points est en général multivaluée.

On cherche à construire des feuilletages de degré 3 tels que l'"application" associée soit une trivolution birationnelle $\mathcal{T}_{\mathcal{F}}$. Notons que pour une telle transformation $\mathcal{T}_{\mathcal{F}}$ la propriété suivante est vérifiée: pour tout point $p$ de $\mathbb{P}^{2}(\mathbb{C}) \backslash \operatorname{Ind}\left(\mathcal{T}_{\mathcal{F}}\right)$, les points $p, \mathcal{T}_{\mathcal{F}}(p)$ et $\mathcal{T}_{\mathcal{F}}^{2}(p)$ sont alignés.

Remarque 5.1. Soit $f: \mathbb{P}^{2}(\mathbb{C})-\rightarrow \mathbb{P}^{2}(\mathbb{C})$ une transformation rationnelle non dégénérée, i.e. génériquement dominante, telle que pour tout $m$ dans $\mathbb{P}^{2}(\mathbb{C})$ les points $m$, $f(m)$ et $f^{2}(m)$ soient alignés; alors

- ou bien $f$ préserve une fibration en droites fibre à fibre;

- ou bien $f$ est birationnelle périodique.

En effet supposons que $f$ ne soit pas périodique. Un argument de Baire montre que si $m$ est un point générique de $\mathbb{P}^{2}(\mathbb{C})$ l'orbite positive 
$\left\{m, f(m), f^{2}(m), \ldots\right\}$ de $m$ sous l'action de $f$ est infinie; alors la droite passant par $m$ et $f(m)$ est invariante par $f$. Il s'en suit que $f$ préserve une infinité de droites et donc une fibration en droites fibre à fibre.

Considérons par exemple les transformations $f_{\kappa, n}=$ $\left(\kappa x+y^{3 n}, \kappa y\right)$ où $\kappa$ est une racine cubique de l'unité et $n$ un entier relatif non nul. On vérifie que les $f_{\kappa, n}$ satisfont tous la condition d'alignement

$$
\operatorname{det}\left(f_{\kappa, n}(m)-m, f_{\kappa, n}^{2}(m)-m\right) \equiv 0 .
$$

Pour $\kappa=1$ les transformations $f_{1, n}$ sont non périodiques et laissent la fibration $y=$ cte invariante. Pour $\kappa=\mathbf{j}$ ou $\mathbf{j}^{2}$ les $f_{\kappa, n}$ sont périodiques de période 3 . Ces dernières transformations ne laissent pas de fibration en droites invariante fibre à fibre.

Soient $\mathcal{F}$ un feuilletage de degré 3 sur $\mathbb{P}^{2}(\mathbb{C})$ et $\mathrm{X}=$ $\mathrm{X}_{1} \frac{\partial}{\partial x}+\mathrm{X}_{2} \frac{\partial}{\partial y}$ un champ de vecteurs polynomial le définissant dans la carte affine $(x, y)$. Soit $m=(x, y)$ un point de $\mathbb{C}^{2}$ non singulier pour $\mathrm{X}$. Si $t$ est un nombre complexe, on note $\mathrm{Y}_{1}(t)$ et $\mathrm{Y}_{2}(t)$ les composantes du champ $\mathrm{X}$ évaluées au point $m+t \mathrm{X}(m)$. Considérons le polynôme $Q(t) \in \mathbb{C}[x, y][t]$ donné par

$$
Q(t)=\mathrm{Y}_{1}(t) \mathrm{X}_{2}-\mathrm{Y}_{2}(t) \mathrm{X}_{1}
$$

Les trois racines $t_{i}$ de $Q$ produisent les points $m+t_{i} \mathrm{X}(m)$ où le feuilletage est tangent à la droite paramétrée par $t \mapsto m+t \mathrm{X}(m)$. Le polynôme $Q$ étant divisible par $t$, il s'écrit $t P(t)$ avec $P(t)=a t^{2}+b t+c$ et $a, b, c$ dans $\mathbb{C}[x, y]$.

On note $\Delta(P)$ (resp. $\Delta(Q))$ le discriminant de $P$ (resp. $Q)$. On vérifie que $\Delta(Q)=c^{2} \Delta(P)$.

Avec les notations précédentes on a l'énoncé suivant.

Proposition 5.2. Supposons que le discriminant $\Delta(P)=$ $b^{2}-4 a c \in \mathbb{C}[x, y]$ soit un carré $s^{2}$ dans $\mathbb{C}[x, y] ;$ notons $r_{1}, r_{2}$ (dans $\mathbb{C}(x, y)$ ) les racines de $P$.

Les applications $\mathcal{T}_{i}=\left(x+r_{i} \mathrm{X}_{1}, y+r_{i} \mathrm{X}_{2}\right)$ sont birationnelles, périodiques de période trois; on a $\mathcal{T}_{1} \circ \mathcal{T}_{2}=\mathrm{id}$.

Démonstration. Les transformations $\mathcal{T}_{1}$ et $\mathcal{T}_{2}$ sont données à réindexation près par

$$
\mathcal{T}_{1}(x, y)=\left(x+\frac{-b+s}{2 a} \mathrm{X}_{1}, y+\frac{-b+s}{2 a} \mathrm{X}_{2}\right)
$$

respectivement

$$
\mathcal{T}_{2}(x, y)=\left(x-\frac{b+s}{2 a} \mathrm{X}_{1}, y-\frac{b+s}{2 a} \mathrm{X}_{2}\right) .
$$

Elles sont rationnelles. Les trois points $(x, y), \mathcal{T}_{1}(x, y)$, $\mathcal{T}_{2}(x, y)$ représentent les trois points de tangence du feuilletage $\mathcal{F}$ avec la droite $(x, y)+\mathbb{C} \cdot \mathrm{X}$ et génériquement ces trois points sont distincts. En particulier $\mathcal{T}_{2}\left(\mathcal{T}_{1}(x, y)\right)$ est l'un de ces trois points de contact; mais il est différent (génériquement) de $\mathcal{T}_{1}(x, y)$, sinon $\mathcal{T}_{2}$ serait l'identité, et de façon analogue différent de $\mathcal{T}_{2}$. Par suite $\mathcal{T}_{2} \circ \mathcal{T}_{1}=\mathrm{id}$ et les $\mathcal{T}_{i}$ sont birationnelles périodiques de période 3 .

Remarque 5.3. Notons que si l'on change $\mathrm{X}$ en $h \cdot \mathrm{X}$, avec $h$ rationnel, la construction produit la même trivolution (remplacer $\mathbb{C}[x, y]$ par $\mathbb{C}(x, y)$ dans la Proposition 5.2): la construction ne dépend que du feuilletage et non du champ le définissant.

Remarque 5.4. Pour un feuilletage $\mathcal{F}$ de degré 3 quelconque on peut associer les transformations multivaluées

$$
\left(x+\frac{-b \pm \sqrt{\Delta}}{2 a} \mathrm{X}_{1}, y+\frac{-b \pm \sqrt{\Delta}}{2 a} \mathrm{X}_{2}\right) \text {. }
$$

Visiblement on pourra associer une trivolution à $\mathcal{F}$ si et seulement si le discriminant est un carré.

Remarque 5.5. On peut d'autre part calculer explicitement les coefficients $a, b$ et $c$. Par exemple on a en carte affine

$$
c=\mathcal{H}(x, y, 1)=\operatorname{det}\left[\begin{array}{ccc}
x & \mathrm{X}_{1} & \mathrm{X}_{1} \frac{\partial \mathrm{X}_{1}}{\partial x}+\mathrm{X}_{2} \frac{\partial \mathrm{X}_{1}}{\partial y} \\
y & \mathrm{X}_{2} & \mathrm{X}_{1} \frac{\partial \mathrm{X}_{2}}{\partial x}+\mathrm{X}_{2} \frac{\partial \mathrm{X}_{2}}{\partial y} \\
1 & 0 & 0
\end{array}\right]
$$

Remarque 5.6. Il est facile de vérifier qu'en général un feuilletage de degré 3 ne produit pas de trivolution. Pour celà considérons le feuilletage de Jouanolou donné par le champ

$$
\left(y^{3}-x^{4}\right) \frac{\partial}{\partial x}+\left(1-x^{3} y\right) \frac{\partial}{\partial y} .
$$

Le polynôme

$$
\begin{aligned}
& \Delta(P)=-3\left(x^{20}-10 x^{16} y^{3}+4 x^{15} y^{7}+10 x^{13} y^{2}+15 x^{12} y^{6}\right. \\
& \quad-10 x^{11} y^{10}-10 x^{10} y-10 x^{9} y^{5}-10 x^{8} y^{9} \\
& +\left(10 y^{13}+4\right) x^{7}+15 x^{6} y^{4}-10 x^{5} y^{8}+15 x^{4} y^{12} \\
& -10\left(y^{3}+y^{16}\right) x^{3}+\left(y^{20}+10 y^{7}\right) x^{2}-10 x y^{11}+y^{2} \\
& \left.+4 y^{15}\right) .
\end{aligned}
$$

n'est pas un carré car sa restriction à $x=0$ n'en est pas un. 
Définition 5.7. Un $d$-tissu singulier (local) est la donnée d'une famille $\left\{\mathcal{F}_{1}, \mathcal{F}_{2}, \ldots, \mathcal{F}_{d}\right\}$ de feuilletages holomorphes singuliers tels qu'en tout point générique $m$ les feuilletages $\mathcal{F}_{i}$ soient deux à deux transverses.

Remarque 5.8. Soit $\mathcal{T}$ une trivolution birationnelle; supposons que génériquement les points $m, \mathcal{T}(m)$ et $\mathcal{T}^{2}(m)$ soient non alignés. On peut associer à $\mathcal{T}$ un 2 -tissu de la façon suivante: les tangentes aux deux feuilles locales passant par $m$ sont les deux droites joignant $m$ à $\mathcal{T}(m)$ et $m$ à $\mathcal{T}^{2}(m)$.

\subsection{Premiers Exemples}

Donnons des exemples de feuilletages $\mathcal{F}$ auxquels on peut associer une trivolution; pour celà on force les polynômes $\Delta(P)$ à être des carrés. Pour tous ces exemples de trivolutions les orbites sont formées de trois points alignés, ce qui n'est évidemment pas le cas général pour une trivolution.

Exemple 5.9. Considérons le feuilletage $\mathcal{F}$ défini par le champ de vecteurs polynomial

$$
x^{3} \frac{\partial}{\partial x}+\frac{\partial}{\partial y} .
$$

Ses feuilles sont les courbes rationnelles $y+\frac{1}{2 x^{2}}=$ cte. On constate que $\mathcal{H}(x, y, z)=-3 x^{5} z^{4}$; on en déduit que $\operatorname{Flex}(\mathcal{F})=\varnothing$.

On remarque que $\Delta(P)=-3 x^{14}$ est effectivement un carré. La trivolution associée à $\mathcal{F}$ est

$$
\mathcal{T}_{\mathcal{F}}=\left(\mathbf{j} x, y+\frac{\mathbf{j}-1}{x^{2}}\right), \quad \mathbf{j}^{3}=1 ;
$$

elle laisse la fibration $x=$ cte invariante et est de degré 3. On a

$$
\begin{aligned}
\operatorname{Ind}\left(\mathcal{T}_{\mathcal{F}}\right) & =\{(0: 1: 0)\}, \\
\operatorname{Exc}\left(\mathcal{T}_{\mathcal{F}}\right) & =\{x=0\}, \\
\operatorname{Fix}\left(\mathcal{T}_{\mathcal{F}}\right) & =\{(1: 0: 0)\} .
\end{aligned}
$$

La transformation $\mathcal{T}_{\mathcal{F}}^{2}$ s'écrit

$$
\left(\mathbf{j}^{2} x, y+\frac{2 \mathbf{j}+1}{\mathbf{j}^{2} x^{2}}\right) ;
$$

elle a même lieux d'indétermination, exceptionnel et ensemble de points fixes que $\mathcal{T}_{\mathcal{F}}$. Finalement $\operatorname{Flex}(\mathcal{F})$ est vide et $\operatorname{Fix}\left(t_{\mathcal{F}}\right)=\operatorname{Fix}\left(\mathcal{T}_{\mathcal{F}}^{2}\right)$ se réduit à un point.

On peut vérifier que

$$
\operatorname{Iso}(\mathcal{F})=\left\{(x, y+\alpha),\left(\beta x, y / \beta^{2}\right) \mid \beta \in \mathbb{C}^{*}, \alpha \in \mathbb{C}\right\} .
$$

Remarquons que $\mathcal{T}_{\mathcal{F}}$ commute à Iso $(\mathcal{F})$; le groupe engendré par Iso $(\mathcal{F})$ et $\mathcal{T}_{\mathcal{F}}$ est une extension triple du groupe affine de la droite.

Notons $f_{0}=y+\frac{1}{2 x^{2}}$ une intégrale première de $\mathcal{F}$. Posons $f_{1}=f_{0} \mathcal{T}_{\mathcal{F}}$ et $f_{2}=f_{0} \mathcal{T}_{\mathcal{F}}^{2}$ les transformés de $f_{0}$ par $\mathcal{T}_{\mathcal{F}}$ et $\mathcal{T}_{\mathcal{F}}^{2}$.

Soit $\mathcal{F}_{i}$ le feuilletage défini par les niveaux de la fonction rationnelle $f_{i}$. Considérons le 3 -tissu $\mathcal{W}=$ $\left(\mathcal{F}_{0}, \mathcal{F}_{1}, \mathcal{F}_{2}\right)$. Si $\left(a_{0}, a_{1}, a_{2}\right)$ est une solution non triviale du système linéaire

$$
a_{0}+a_{1}+a_{2}=0, \quad a_{0} \mathbf{j}^{2}+\left(3-2 \mathbf{j}^{2}\right) a_{1}+(5 \mathbf{j}+2) a_{2}=0,
$$

alors $a_{0} f_{0}+a_{1} f_{1}+a_{2} f_{2}=0$. En d'autres termes le tissu $\mathcal{W}=\left(\mathcal{F}_{0}, \mathcal{F}_{1}, \mathcal{F}_{2}\right)$ est hexagonal [Beauville 80]. C'est un phénomène que nous allons retrouver de façon récurrente dans les exemples qui suivent. Rappelons ce qu'est un tissu hexagonal. Soit $m$ un point générique; en $m$ le feuilletage $\mathcal{F}_{i}$ est défini par les niveaux d'une certaine submersion locale. Lorsque l'on peut trouver trois telles submersions $f_{i}$ définissant $\mathcal{F}_{i}$ et satisfaisant la relation (abélienne) $f_{1}+f_{2}+f_{3}=0$ on dit que le tissu est hexagonal. On renvoie à [Beauville 80] pour la justification de la terminologie.

Exemple 5.10. Si $\mathcal{F}$ est le feuilletage décrit par

$$
\left(x^{3}-1\right) \frac{\partial}{\partial x}+\frac{\partial}{\partial y},
$$

le polynôme $\Delta(P)$ vaut $-3 x^{2}\left(x^{3}-1\right)^{4}$; comme c'est un carré, on peut associer à $\mathcal{F}$ une trivolution

$$
\mathcal{T}_{\mathcal{F}}=\left(\mathbf{j} x, \frac{x^{3} y-y+(\mathbf{j}-1) x}{x^{3}-1}\right)
$$

pour laquelle on a

$$
\begin{aligned}
\operatorname{Ind}\left(\mathcal{T}_{\mathcal{F}}\right) & =\{(0: 1: 0)\} \\
\operatorname{Exc}\left(\mathcal{T}_{\mathcal{F}}\right) & =\{x-z=0\} \cup\{x-\mathbf{j} z=0\} \cup\left\{x-\mathbf{j}^{2} z=0\right\}, \\
\operatorname{Fix}\left(\mathcal{T}_{\mathcal{F}}\right) & =\{(1: 0: 0)\} \cup\{x=0\} .
\end{aligned}
$$

Son carré s'écrit

$$
\mathcal{T}_{\mathcal{F}}^{2}=\left(\mathbf{j}^{2} x, \frac{x^{3} y-y+\left(\mathbf{j}^{2}-1\right) x}{x^{3}-1}\right)
$$

et

$$
\begin{aligned}
\operatorname{Ind}\left(\mathcal{T}_{\mathcal{F}}^{2}\right) & =\operatorname{Ind}\left(\mathcal{T}_{\mathcal{F}}\right), \\
\operatorname{Exc}\left(\mathcal{T}_{\mathcal{F}}^{2}\right) & =\operatorname{Exc}\left(\mathcal{T}_{\mathcal{F}}\right), \\
\operatorname{Fix}\left(\mathcal{T}_{\mathcal{F}}^{2}\right) & =\operatorname{Fix}\left(\mathcal{T}_{\mathcal{F}}\right) .
\end{aligned}
$$


Un calcul montre que

$$
\mathcal{H}(x, y, z)=-3 x^{2} z^{4}(x-z)(x-\mathbf{j} z)\left(x-\mathbf{j}^{2} z\right) .
$$

Les droites $z=0$ et $x=z$ sont invariantes par $\mathcal{F}$ par contre celle d'équation $x=0$ (resp. $x-\mathbf{j} z=0$, resp. $\left.x-\mathbf{j}^{2} z=0\right)$ ne l'est pas; par suite

$$
\operatorname{Flex}(\mathcal{F})=\{x=0\} \cup\{x-\mathbf{j} z=0\} \cup\left\{x-\mathbf{j}^{2} z=0\right\} .
$$

Les droites $x-\mathbf{j} z=0$ et $x-\mathbf{j}^{2} z=0$ sont contractées par $\mathcal{T}_{\mathcal{F}}$ et $\mathcal{T}_{\mathcal{F}}^{2}$, la droite $x=0$ est l'unique courbe de points fixes de $\mathcal{T}_{\mathcal{F}}$ (resp. $\mathcal{T}_{\mathcal{F}}^{2}$ ); autrement dit les courbes de $\operatorname{Flex}(\mathcal{F})$ sont soit contractées par $\mathcal{T}_{\mathcal{F}}$, soit fixées par $\mathcal{T}_{\mathcal{F}}$.

Alors que le feuilletage précédent possède une intégrale première rationnelle celui-ci a une intégrale première de type Liouville

$$
3 y-\ln (x-1)-\mathbf{j} \ln (x-\mathbf{j})-\mathbf{j}^{2} \ln \left(x-\mathbf{j}^{2}\right) .
$$

Notons $f_{0}$ cette intégrale première et posons $f_{1}=$ $f_{0} \mathcal{T}_{\mathcal{F}}, f_{2}=f_{0} \mathcal{T}_{\mathcal{F}}^{2}$. Comme précédemment le 3 -tissu $\mathcal{W}=\left(\mathcal{F}_{0}, \mathcal{F}_{1}, \mathcal{F}_{2}\right.$ ) (où les $\mathcal{F}_{i}$ sont les feuilletages par les niveaux des $f_{i}$ ) est hexagonal.

Remarque 5.11. Le feuilletage associé au champ de vecteurs

$$
\mathrm{X}_{\varepsilon}=\left(x^{3}-\varepsilon\right) \frac{\partial}{\partial x}+\frac{\partial}{\partial y}
$$

est conjugué pour $\varepsilon$ non nul au champ $\mathrm{X}_{1}$ considéré dans l'Exemple 5.10 par la transformation linéaire $\left(\varepsilon^{1 / 3} x, \varepsilon^{-2 / 3} y\right)$. Lorsque $\varepsilon$ tend vers 0 , le feuilletage et son involution

$$
\left(\mathbf{j} x, \frac{x^{3} y-\varepsilon y+(\mathbf{j}-1) x}{x^{3}-\varepsilon}\right)
$$

dégénèrent sur ceux de l'Exemple 5.9; on note une chute de degré de la trivolution pour $\varepsilon=0$.

Exemple 5.12. Considérons le feuilletage $\mathcal{F}$ d'intégrale première

$$
y-\frac{1}{3} \ln x+\frac{1}{x}+\frac{1}{2 x^{2}} ;
$$

il est aussi défini par

$$
x^{3} \frac{\partial}{\partial x}+\left(1+x+\frac{1}{3} x^{2}\right) \frac{\partial}{\partial y} .
$$

Comme $\Delta(P)=-\frac{1}{3} x^{14}(x+3)^{2}$, on associe à $\mathcal{F}$ la trivolution

$$
\mathcal{T}_{\mathcal{F}}=\left(\frac{3 \mathbf{j} x}{(1-\mathbf{j}) x+3}, \frac{3 x^{2} y-x^{2}+(\mathbf{j}-4) x+3(\mathbf{j}-1)}{3 x^{2}}\right)
$$

qui préserve la fibration $x=$ cte. On a

$$
\begin{aligned}
\operatorname{Ind}\left(\mathcal{T}_{\mathcal{F}}\right) & =\{(0: 1: 0),(1: 0: 0)\} \\
\operatorname{Fix}\left(\mathcal{T}_{\mathcal{F}}\right) & =\{x+3 z=0\} \\
\operatorname{Exc}\left(\mathcal{T}_{\mathcal{F}}\right) & =\{x=0\} \cup\{z=0\} \cup\{(\mathbf{j}-1) x-3 z=0\} .
\end{aligned}
$$

On constate que

$$
\begin{aligned}
\mathcal{T}_{\mathcal{F}}^{2} & =\left(\frac{3(\mathbf{j}-1)^{4} x}{16((\mathbf{j}+2) x+3)},\right. \\
& \left.\frac{3\left(3 \mathbf{i} \sqrt{3}+(4+5 \mathbf{j}) x+(1+\mathbf{j}) x^{2}-3(1+\mathbf{j}) x^{2} y\right)}{(\mathbf{j}-1)^{4} x^{2}}\right) ;
\end{aligned}
$$

on vérifie que

$$
\begin{aligned}
\operatorname{Ind}\left(\mathcal{T}_{\mathcal{F}}^{2}\right) & =\operatorname{Ind}\left(\mathcal{T}_{\mathcal{F}}\right), \\
\operatorname{Fix}\left(\mathcal{T}_{\mathcal{F}}^{2}\right) & =\operatorname{Fix}\left(\mathcal{T}_{\mathcal{F}}\right), \\
\operatorname{Exc}\left(\mathcal{T}_{\mathcal{F}}^{2}\right) & =\{x=0\} \cup\{z=0\} \cup\{(\mathbf{j}+2) x+3 z=0\} .
\end{aligned}
$$

À l'inverse de l'exemple précédent les ensembles exceptionnels de $\mathcal{T}_{\mathcal{F}}$ et $\mathcal{T}_{\mathcal{F}}^{2}$ diffèrent. Un calcul montre que $\mathcal{H}(x, y, z)=-\frac{1}{3} x^{5} z^{2}(x+3 z)^{2}$. Les droites $x=0$ et $z=0$ sont invariantes par $\mathcal{F}$ alors que celle d'équation $x+3 z=0$ ne l'est pas; par suite $\operatorname{Flex}(\mathcal{F})=\{x+3 z=0\}$ et $\operatorname{Flex}(\mathcal{F})=\operatorname{Fix}\left(\mathcal{T}_{\mathcal{F}}\right)=\operatorname{Fix}\left(\mathcal{T}_{\mathcal{F}}^{2}\right)$.

En conjuguant $\mathcal{F}$ par $\left(\varepsilon x, \frac{y}{\varepsilon^{3}}\right)$ on obtient la famille de feuilletages $\left(\mathcal{F}_{\varepsilon}\right)$ décrite par les champs

$$
x^{3} \frac{\partial}{\partial x}+\left(1+\varepsilon x+\frac{\varepsilon^{2}}{3} x^{2}\right) \frac{\partial}{\partial y}, \quad \varepsilon \neq 0
$$

à laquelle on peut associer la famille de trivolutions

$$
\begin{aligned}
\mathcal{T}_{\mathcal{F}_{\varepsilon}}= & \frac{3 \mathbf{j} x}{(1-\mathbf{j}) \varepsilon x+3}, \\
& \left.\frac{3 x^{2} y-\varepsilon^{2} x^{2}+(\mathbf{j}-4) \varepsilon x+3(\mathbf{j}-1)}{3 x^{2}}\right) .
\end{aligned}
$$

La famille $\left(\mathcal{T}_{\mathcal{F}_{\varepsilon}}\right)$ de transformations de degré 4 pour $\varepsilon \neq 0$ dégénère encore pour $\varepsilon=0$ sur la trivolution de degré 3 traitée dans l'Exemple 5.9.

\subsection{Feuilletages Homogènes}

On va s'intéresser à une famille très spéciale de feuilletages: les feuilletages homogènes génériques de degré 3. À conjugaison près un feuilletage homogène générique de degré 3 à l'origine de $\mathbb{C}^{2} \subset \mathbb{P}^{2}(\mathbb{C})$ est donné par une 1-forme fermée rationnelle du type suivant

$$
\frac{\mathrm{d} x}{x}+\lambda \frac{\mathrm{d} y}{y}+\mu \frac{\mathrm{d}(y-x)}{y-x}+\nu \frac{\mathrm{d}(y-\alpha x)}{y-\alpha x}
$$


avec

$$
\alpha \lambda \mu \nu(1+\lambda+\mu+\nu) \neq 0, \quad \alpha \neq 1
$$

si $\lambda+\mu+\nu+1=0$ le feuilletage correspondant est de degré 0 . Il possède l'intégrale première multivaluée $x y^{\lambda}(y-$ $x)^{\mu}(y-\alpha x)^{\nu}$. On note $\mathcal{F}(\alpha ; \lambda, \mu, \nu)$ un tel feuilletage. Il $\mathrm{y}$ a donc quatre paramètres, trois de type résidus $\lambda, \mu, \nu$ et $\alpha$ qui positionne les droites invariantes.

Définition 5.13. Le quadruplet $(\alpha, \lambda, \mu, \nu)$ est admissible si $\alpha \lambda \mu \nu(\lambda+\mu+\nu+1) \neq 0$ et $\alpha \neq 1$.

Le champ de vecteurs homogène

$$
\begin{aligned}
& -x(\lambda(y-x)(y-\alpha x)+\mu y(y-\alpha x)+\nu y(y-x)) \frac{\partial}{\partial x} \\
& +y((y-x)(y-\alpha x)-\mu x(y-\alpha x)-\nu \alpha x(y-x)) \frac{\partial}{\partial y}
\end{aligned}
$$

définit aussi le feuilletage $\mathcal{F}$ car est dans le noyau de la forme fermée précédente. Un tel $\mathcal{F}$ est invariant par homothétie. En particulier si $\mathcal{F}$ induit une trivolution $\mathcal{T}_{\mathcal{F}}$, celle-ci commute à toutes les homothéthies. Un calcul élémentaire montre que $\mathcal{T}_{\mathcal{F}}$ préserve la fibration radiale $y / x=$ constante: $\mathcal{T}_{\mathcal{F}}$ est donc de Jonquières.

Commençons par quelques exemples simples.

Exemple 5.14. Considérons le feuilletage $\mathcal{F}$ donné par le champ de vecteurs

$$
y^{3} \frac{\partial}{\partial x}+x^{3} \frac{\partial}{\partial y}
$$

Les feuilles de $\mathcal{F}$ sont les niveaux de $y^{4}-x^{4}$ et sont donc des courbes de genre 3 . Un calcul montre que

$$
\mathcal{H}(x, y, z)=3 x^{2} y^{2} z(x+\mathbf{i} y)(x-\mathbf{i} y)(y-x)(y+x) .
$$

Puisque les droites $x-y=0, x+y=0, x-\mathbf{i} y=0$, $x+\mathbf{i} y=0$ sont invariantes par $\mathcal{F}$, on a $\operatorname{Flex}(\mathcal{F})=\{x=$ $0\} \cup\{y=0\} \cup\{z=0\}$.

Le polynôme

$$
\Delta(P)=3 x^{2} y^{2}(x-y)^{4}(x+y)^{4}(x+\mathbf{i} y)^{4}(x-\mathbf{i} y)^{4}
$$

étant un carré, est associée à $\mathcal{F}$ la trivolution de Jonquières de degré 5 donnée par

$$
\mathcal{T}_{\mathcal{F}}=\left(\frac{x\left(x^{4}-y^{4}\right)}{x^{4}-\mathbf{j} y^{4}}, \frac{\mathbf{j} y\left(x^{4}-y^{4}\right)}{x^{4}-\mathbf{j} y^{4}}\right) .
$$

L'ensemble d'indétermination de $\mathcal{T}_{\mathcal{F}}$

$\operatorname{Ind}\left(\mathcal{T}_{\mathcal{F}}\right)=\{(1: 1: 0),(1:-1: 0),(\mathbf{i}: 1: 0),(-\mathbf{i}: 1: 0)$, $(0: 0: 1)\}$ est le lieu singulier de $\mathcal{F}$ et $\operatorname{Fix}\left(\mathcal{T}_{\mathcal{F}}\right)=\{x=0\} \cup\{y=0\} \cup$ $\{z=0\}$ coïncide avec l'ensemble des points d'inflexion de $\mathcal{F}$. L'ensemble exceptionnel de $\mathcal{F}$

$$
\begin{aligned}
\operatorname{Exc}\left(\mathcal{T}_{\mathcal{F}}\right)= & \{x+y=0\} \cup\{x-y=0\} \cup\{y-\mathbf{i} x=0\} \\
& \cup\{y+\mathbf{i} x=0\} \cup\{x-\mathbf{j} y=0\} \cup\{x+\mathbf{j} y=0\} \\
& \cup\{x-\mathbf{i} \mathbf{j} y=0\} \cup\{x+\mathbf{i} \mathbf{j} y=0\}
\end{aligned}
$$

est constitué de huit droites.

On vérifie que

$$
\mathcal{T}_{\mathcal{F}}^{2}=\left(\frac{x\left(x^{4}-y^{4}\right)}{x^{4}-\mathbf{j}^{2} y^{4}}, \frac{\mathbf{j}^{2} y\left(x^{4}-y^{4}\right)}{x^{4}-\mathbf{j}^{2} y^{4}}\right)
$$

a même lieu d'indétermination et même ensemble de points fixes que $\mathcal{T}_{\mathcal{F}}$. Les ensembles exceptionnels diffèrent puisque

$$
\begin{aligned}
\operatorname{Exc}\left(\mathcal{T}_{\mathcal{F}}^{2}\right)= & \{x+y=0\} \cup\{x-y=0\} \cup\{y-\mathbf{i} x=0\} \\
& \cup\{y+\mathbf{i} x=0\} \cup\left\{x-\mathbf{j}^{2} y=0\right\} \\
& \cup\left\{x+\mathbf{j}^{2} y=0\right\} \cup\left\{x-\mathbf{i j}^{2} y=0\right\} \\
& \cup\left\{x+\mathbf{i j}^{2} y=0\right\} .
\end{aligned}
$$

Posons $f_{0}=x^{4}-y^{4}$. Faisons agir $\mathcal{T}_{\mathcal{F}}$ et $\mathcal{T}_{\mathcal{F}}^{2}$ sur $\mathcal{F}$ pour obtenir les feuilletages $\mathcal{F}_{1}$ et $\mathcal{F}_{2}$ définis par les fonctions rationnelles $f_{1}=f_{0} \mathcal{T}_{\mathcal{F}}$ et $f_{2}=f_{0} \mathcal{T}_{\mathcal{F}}^{2}$. Une fois de plus considérons le 3 -tissu $\mathcal{W}$ associé aux trois fonctions rationnelles $f_{0}, f_{1}, f_{2}$; en un point générique il est aussi donné par

$$
\begin{aligned}
& F_{0}=f_{0}^{-1 / 3}=\frac{x^{4}-y^{4}}{\left(x^{4}-y^{4}\right)^{4 / 3}}, \\
& F_{1}=f_{1}^{-1 / 3}=\frac{x^{4}-\mathbf{j} y^{4}}{\left(x^{4}-y^{4}\right)^{4 / 3}} \\
& F_{2}=f_{2}^{-1 / 3}=\frac{x^{4}-\mathbf{j}^{2} y^{4}}{\left(x^{4}-y^{4}\right)^{4 / 3}} .
\end{aligned}
$$

Soit $\left(a_{0}, a_{1}, a_{2}\right)$ une solution non triviale du système linéaire

$$
a_{0}+a_{1}+a_{2}=0, \quad a_{0}+\mathbf{j} a_{1}+\mathbf{j}^{2} a_{2}=0 ;
$$

alors $a_{0} F_{0}+a_{1} F_{1}+a_{2} F_{2}=0$ : le tissu $\mathcal{W}$ est hexagonal.

On obtient ainsi parmi les feuilletages homogènes génériques l'exemple d'un feuilletage "hamiltonien" auquel est associé une trivolution. On peut se demander si c'est le seul. La réponse est donnée par l'énoncé suivant.

Proposition 5.15. On peut associer une trivolution à $\mathcal{F}=$ $\mathcal{F}(\alpha ; 1,1,1)$ si et seulement si $\alpha$ vaut $-1,2$ ou $1 / 2 ; \dot{a}$ conjugaison linéaire près on est dans la situation décrite dans l'Exemple 5.14. 
Démonstration. Reprenons les notations introduites au §5.2. L'application associée à $\mathcal{F}$ est birationnelle si $\Delta(P)$ est un carré. Or $\Delta(P)$ s'écrit $1024 x^{4} y^{4}\left(x^{2}-(1+\alpha) x y+\right.$ $\left.\alpha y^{2}\right)^{4} R(x, y)$ où $R(x, y)$ désigne

$$
\begin{aligned}
& \left(1-\alpha+\alpha^{2}\right) x^{4}-4(1-\alpha)^{2}(1+\alpha) x^{3} y \\
& \quad-2\left(\alpha^{3}+\alpha^{2}+\alpha-2\right) x^{2} y^{2}-4 \alpha(1-\alpha)^{2}(1+\alpha) x y^{3} \\
& \quad+\alpha^{2}\left(1-\alpha+\alpha^{2}\right) y^{4}
\end{aligned}
$$

donc $\Delta(P)$ est un carré si et seulement si $R$ en est un. Un calcul montre que $R$ est un carré si et seulement si $\alpha$ prend les valeurs $-1,1 / 2$ ou 2 .

Posons $h_{\alpha}=x y(y-x)(y-\alpha x)$. Les polynômes $h_{-1}$, $h_{1 / 2}$ et $h_{2}$ sont linéairement conjugués; de plus, quitte à faire le changement de variables

$$
\left(\kappa x+\kappa\left(4 \kappa^{2}-1\right) y, \kappa x+\kappa\left(1-4 \kappa^{2}\right) y\right),
$$

où

$$
\kappa=2^{-3 / 4} \exp \left(\frac{i \pi}{8}\right)
$$

on constate que $y^{4}-x^{4}$ s'écrit $x y(x-y)(x+y)$.

Remarque 5.16. La configuration des quatre droites qui apparaissent dans la Proposition 5.15 est la configuration spéciale, celle qui possède un groupe d'automorphismes à 12 éléments.

La Proposition 5.15 possède de nombreuses généralisations, en voici une. Le schéma de preuve est exactement le même que celui de la Proposition 5.15, les calculs étant un petit peu plus pénibles.

Proposition 5.17. On considère $\mathcal{F}$ un élément de la famille $\mathcal{F}(\alpha ; 1, \mu, \mu)$. On peut associer à $\mathcal{F}$ une trivolution $\mathcal{T}_{\mathcal{F}}$ si et seulement si $\mu$ vaut 1 et $\alpha$ vaut $-1,2$ ou $1 / 2$; ̀̀ conjugaison linéaire près on est dans la situation de l'Exemple 5.14.

Exemple 5.18. Soit $\mathcal{F}$ le feuilletage donné par le champ de vecteurs

$$
x^{3} \frac{\partial}{\partial x}+y^{3} \frac{\partial}{\partial y} .
$$

On remarque que $x^{2} y^{2}(x-y)^{-1}(x+y)^{-1}$ est une intégrale première rationnelle de $\mathcal{F}$; ses feuilles sont des quartiques ayant deux singularités ordinaires, donc des courbes elliptiques. On vérifie que $\mathcal{H}(x, y, z)=3 x^{3} y^{3} z(x+y)(y-x)$; on en déduit que $\operatorname{Flex}(\mathcal{F})=\varnothing$. On constate que
$\Delta(P)=3 x^{6} y^{6}(x+y)^{4}(y-x)^{4}$ est un carré. La trivolution associée à $\mathcal{F}$ s'écrit

$$
\mathcal{T}_{\mathcal{F}}=\left(\frac{\mathbf{j} x\left(x^{2}-y^{2}\right)}{x^{2}-\mathbf{j} y^{2}}, \frac{y\left(x^{2}-y^{2}\right)}{x^{2}-\mathbf{j} y^{2}}\right) ;
$$

c'est encore une transformation de Jonquières qui est de degré 3. Son ensemble exceptionnel

$$
\begin{aligned}
\operatorname{Exc}\left(\mathcal{T}_{\mathcal{F}}\right)= & \{x-y=0\} \cup\{x+y=0\} \cup\{\mathbf{j} x-y=0\} \\
& \cup\{\mathbf{j} x+y=0\}
\end{aligned}
$$

est constitué de quatre droites dont deux sont invariantes par le feuilletage. On remarque que

$$
\mathcal{T}_{\mathcal{F}}^{2}=\left(\frac{x\left(x^{2}-y^{2}\right)}{\mathbf{j} x^{2}-y^{2}}, \frac{\mathbf{j} y\left(x^{2}-y^{2}\right)}{\mathbf{j} x^{2}-y^{2}}\right) .
$$

L'ensemble exceptionnel de $\mathcal{T}_{\mathcal{F}}^{2}$,

$$
\begin{aligned}
\operatorname{Exc}\left(\mathcal{T}_{\mathcal{F}}^{2}\right)= & \{x-y=0\} \cup\{x+y=0\} \cup\{\mathbf{j} y-x=0\} \\
\cup\{\mathbf{j} y+x=0\} &
\end{aligned}
$$

est encore formé des deux mêmes droites invariantes auxquelles s'ajoutent deux nouvelles droites. C'est un exemple où $\operatorname{Flex}(\mathcal{F})$ est vide et où la trivolution a seulement des points fixes isolés.

Posons $f_{0}=\frac{x^{2} y^{2}}{x^{2}-y^{2}}$ et $\mathcal{F}_{0}=\mathcal{F}$. Faisons agir $\mathcal{T}_{\mathcal{F}}$ et $\mathcal{T}_{\mathcal{F}}^{2}$ sur $\mathcal{F}$ pour obtenir les feuilletages $\mathcal{F}_{1}$ et $\mathcal{F}_{2}$ définis par les fonctions rationnelles $f_{1}=f_{0} \mathcal{T}_{\mathcal{F}}$ et $f_{2}=f_{0} \mathcal{T}_{\mathcal{F}}^{2}$. Considérons le 3 -tissu $\mathcal{W}=\left(\mathcal{F}_{0}, \mathcal{F}_{1}, \mathcal{F}_{2}\right)$; en un point générique il est aussi donné par

$$
\begin{aligned}
& F_{0}=f_{0}^{-1 / 3}=\frac{x^{2}-y^{2}}{\left(x y\left(x^{2}-y^{2}\right)\right)^{2 / 3}}, \\
& F_{1}=f_{1}^{-1 / 3}=\frac{x^{2}-\mathbf{j} y^{2}}{\left(x y\left(x^{2}-y^{2}\right)\right)^{2 / 3}}, \\
& F_{2}=f_{2}^{-1 / 3}=\frac{x^{2}-\mathbf{j}^{2} y^{2}}{\left(x y\left(x^{2}-y^{2}\right)\right)^{2 / 3}} .
\end{aligned}
$$

Soient $\left(a_{0}, a_{1}, a_{2}\right)$ une solution non triviale du système linéaire

$$
a_{0}+a_{1}+a_{2}=0, \quad a_{0}+\mathbf{j} a_{1}+\mathbf{j}^{2} a_{2}=0
$$

alors $a_{0} F_{0}+a_{1} F_{1}+a_{2} F_{2}=0$ : le tissu $\mathcal{W}$ est hexagonal.

La Proposition 5.15 suggère que la configuration $\alpha \in$ $\{-1,2,1 / 2\}$ joue un rôle très spécial.

Venons-en au cas général. On va décrire dans l'espace des paramètres $\left\{(\alpha, \lambda, \mu, \nu) \in\left(\mathbb{C}^{*}\right)^{4} \mid \alpha \neq 1\right\}$ les feuilletages $\mathcal{F}(\alpha ; \lambda, \mu, \nu)$ auxquels est associée une trivolution. Introduisons $\Lambda$ le projectivisé de l'espace des polynômes 
homogènes de degré 4 en 2 variables; on remarque que $\Lambda \simeq \mathbb{P}^{4}(\mathbb{C})$. Posons

$$
\Theta=\{T \in \Lambda \mid T \text { est un carré }\} \text {. }
$$

Un élément $T=\tau_{1} x^{4}+\tau_{2} x^{3} y+\tau_{3} x^{2} y^{2}+\tau_{4} x y^{3}+\tau_{5} y^{4}$ appartient à $\Theta$ si et seulement s'il existe des complexes $A, B$ et $C$ tels que $T=\left(A x^{2}+B x y+C y^{2}\right)^{2}$; autrement dit si et seulement si

$$
\begin{aligned}
& \tau_{1}=A^{2}, \quad \tau_{2}=2 A B, \quad \tau_{3}=B^{2}+2 A C, \quad \tau_{4}=2 B C, \\
& \tau_{5}=C^{2}
\end{aligned}
$$

L'application

$$
\Psi:(A, B, C) \mapsto\left(A^{2}, 2 A B, B^{2}+2 A C, 2 B C, C^{2}\right)
$$

induit une application holomorphe, en fait un plongement de $\mathbb{P}^{2}(\mathbb{C})$ dans $\mathbb{P}^{4}(\mathbb{C})$ noté encore $\Psi$. Son image, qui est $\Theta$, est en fait une projection de la surface de Veronese standard de $\mathbb{P}^{5}(\mathbb{C})$ sur $\mathbb{P}^{4}(\mathbb{C})$. On peut évidemment donner l'idéal de définition de $\Theta$. Toutefois pour les calculs pratiques il est plus commode d'utiliser la présentation ensembliste suivante. On découpe l'espace $\mathbb{P}^{4}(\mathbb{C})$ comme l'union de l'hyperplan $\tau_{5}=0$ et de l'ouvert (carte affine) $\tau_{5}=1$ et nous allons décrire $\Theta$ relativement à ce découpage. Pour $T$ comme ci-dessus on note encore $T=\left(\tau_{1}: \cdots: \tau_{5}\right)$. Remarquons que $\Psi$ envoie la carte affine $C=1$ de $\mathbb{P}^{2}(\mathbb{C})$ dans la carte affine $\tau_{5}=1 \mathrm{de} \mathbb{P}^{4}(\mathbb{C})$. Ainsi si $T=\left(\tau_{1}: \cdots: \tau_{4}: 0\right)$ est dans $\Psi(\{C=0\})$ on a

(i) $\tau_{4}=\tau_{5}=0$ et $4 \tau_{1} \tau_{3}-\tau_{2}^{2}=0$.

Maintenant si $T=\left(\tau_{1}: \cdots: \tau_{4}: 1\right)$ est dans $\Psi(\{C=1\})$ on vérifie que lorsque $\tau_{4}=0$ on a

(ii) $\tau_{5}=1, \tau_{2}=\tau_{4}=\tau_{3}^{2}-4 \tau_{1}=0$

et que pour $\tau_{4} \neq 0$ les $\tau_{i}$ vérifient

(iii) $\tau_{5}=1, \tau_{4} \neq 0, \tau_{4}^{2} \tau_{1}-\tau_{2}^{2}=4 \tau_{3} \tau_{4}-\tau_{4}^{3}-8 \tau_{2}=0$.

On remarque que l'annulation de $\tau_{4}$ dans (iii) ne produit pas (ii).

Soit donc $\mathcal{F}=\mathcal{F}(\alpha ; \lambda, \mu, \nu)$, le quadruplet $(\alpha, \lambda, \mu, \nu)$ étant admissible. Avec les notations précédentes on obtient

$\Delta(P)=(\lambda+\nu+\mu+1)^{4} x^{4} y^{4}\left(y^{2}-(\alpha+1) x y+\alpha x^{2}\right)^{4} R(x, y)$

où $R(x, y)$ désigne le polynôme $r_{1} x^{4}+r_{2} x^{3} y+r_{3} x^{2} y^{2}+$ $r_{4} x y^{3}+r_{5} y^{4}$ avec

$$
\begin{aligned}
& r_{1}=\lambda^{2} \alpha^{2}\left(2 \alpha \nu \mu+\alpha^{2}+2 \alpha^{2} \nu+\alpha^{2} \nu^{2}\right. \\
&\left.-2 \alpha-2 \alpha \mu-2 \alpha \nu+1+2 \mu+\mu^{2}\right),
\end{aligned}
$$

$$
\begin{aligned}
r_{2}= & -2 \lambda \alpha\left(\lambda \alpha \nu \mu+\lambda \alpha^{2} \mu \nu-3 \alpha^{2} \mu \nu+\nu \mu^{2}+\lambda \mu^{2}-\lambda \alpha \nu\right. \\
& +\alpha \nu^{2} \mu-2 \alpha \nu \mu^{2}+\alpha^{2} \nu \mu^{2}-3 \alpha \nu \mu-\lambda \alpha \mu-\lambda \alpha^{2} \mu \\
& +2 \nu \mu-\alpha^{2} \mu^{2}+\lambda+\nu+2 \mu \lambda-\lambda \alpha^{2}-\lambda \alpha+\lambda \alpha^{3} \\
& +\nu^{2} \mu \alpha^{3}+2 \nu \mu \alpha^{3}-\alpha \nu-\alpha^{2} \mu+\mu \alpha^{3}-\lambda \nu \alpha^{2} \\
& \left.-2 \mu \nu^{2} \alpha^{2}-\nu^{2} \alpha+2 \lambda \nu \alpha^{3}+\lambda \nu^{2} \alpha^{3}\right) \\
& \\
r_{3}= & 2 \mu \nu^{2}-4 \lambda \alpha^{2} \mu^{2}-4 \lambda \alpha \nu \mu^{2}-2 \lambda \alpha \nu \mu+2 \nu^{2} \lambda \alpha^{2} \mu \\
& -12 \lambda \alpha^{2} \mu \nu+2 \lambda^{2} \nu \alpha^{2} \mu+2 \lambda \nu \alpha^{2} \mu^{2}+4 \lambda \nu \mu \alpha^{4} \\
& -4 \lambda \nu^{2} \mu \alpha^{3}+2 \lambda \nu^{2} \mu \alpha^{4}+4 \lambda \nu \mu+6 \nu^{2} \alpha^{2} \mu^{2} \\
& -4 \lambda^{2} \alpha^{2} \nu-4 \lambda^{2} \alpha^{2} \mu-4 \alpha \nu^{2} \mu+2 \alpha^{2} \nu \mu^{2}-4 \lambda \alpha^{2} \mu \\
& +2 \lambda^{2} \alpha \mu+2 \mu \lambda \alpha^{4}+2 \alpha^{2} \mu \nu-2 \lambda \nu \mu \alpha^{3}+2 \lambda \nu \mu^{2} \\
& +2 \lambda \alpha \nu-4 \alpha \mu^{2} \nu^{2}+2 \lambda^{2} \mu+\lambda^{2} \mu^{2}+2 \lambda^{2} \alpha-6 \lambda^{2} \alpha^{2} \\
& +\nu^{2}+2 \lambda \nu+\lambda^{2}+\mu^{2} \alpha^{4}+\lambda^{2} \alpha^{4}+2 \lambda^{2} \alpha^{3}-4 \mu^{2} \nu^{2} \alpha^{3} \\
& +2 \lambda^{2} \nu \alpha^{4}+\mu^{2} \nu^{2}-4 \lambda \nu \alpha^{2}-4 \nu \mu^{2} \alpha^{3}+2 \mu \nu^{2} \alpha^{2} \\
& -4 \lambda \nu^{2} \alpha^{2}+2 \lambda^{2} \nu \alpha^{3}+2 \mu \lambda \alpha^{3}+\mu^{2} \nu^{2} \alpha^{4}+2 \nu \mu^{2} \alpha^{4} \\
& +\lambda^{2} \nu^{2} \alpha^{4},
\end{aligned}
$$

$$
\begin{aligned}
r_{4}= & -2\left(\mu \nu^{2}-3 \lambda \alpha \nu \mu-3 \lambda \alpha^{2} \mu \nu+\alpha^{2} \mu \nu+2 \lambda \nu \mu \alpha^{3}\right. \\
& -\lambda \alpha \nu+2 \lambda \nu \mu-\lambda^{2} \alpha^{2} \nu-2 \alpha \nu^{2} \mu+\alpha \nu \mu^{2}-2 \alpha^{2} \nu \mu^{2} \\
& +\alpha \nu \mu-\lambda \alpha \mu-\lambda \alpha \mu^{2}-\lambda \alpha^{2} \mu+\mu^{2} \alpha^{3}+\lambda^{2} \alpha^{3} \\
& -\lambda \nu \alpha^{2}+\nu \mu^{2} \alpha^{3}+\mu \nu^{2} \alpha^{2}-\lambda \nu^{2} \alpha^{2}+\lambda^{2} \nu \alpha^{3} \\
& +2 \mu \lambda \alpha^{3}-\lambda^{2} \alpha \mu+\lambda^{2} \mu-\lambda^{2} \alpha-\lambda^{2} \alpha^{2}+\nu^{2} \\
& \left.+2 \lambda \nu+\lambda^{2}\right),
\end{aligned}
$$

$$
\begin{aligned}
r_{5}= & \lambda^{2}+\lambda^{2} \alpha^{2}-2 \lambda^{2} \alpha+2 \lambda \alpha^{2} \mu+2 \lambda \nu-2 \lambda \alpha \nu \\
& -2 \lambda \alpha \mu+2 \alpha \nu \mu+\nu^{2}+\alpha^{2} \mu^{2} .
\end{aligned}
$$

Considérons l'application $f$ définie par

$$
\mathbb{C}^{4} \rightarrow \mathbb{P}^{4}(\mathbb{C}), \quad(\alpha, \lambda, \mu, \nu) \mapsto\left(r_{1}: r_{2}: r_{3}: r_{4}: r_{5}\right)
$$

L'ensemble $f^{-1}(\Theta)$ donne les paramètres $(\alpha, \lambda, \mu, \nu)$ pour lesquels le discriminant associé est un carré; cet ensemble s'identifie donc à celui des feuilletages homogènes $\mathcal{F}(\alpha ; \lambda, \mu, \nu)$ auxquels on peut associer une trivolution. On va décrire $f^{-1}(\Theta)$ près du point spécial $(-1,1,1,1)$. Plaçons-nous dans la carte $\tau_{5}=1$. La matrice jacobienne de $f$ au point $(-1,1,1,1)$ est

$$
\operatorname{Jac}(f)_{(-1,1,1,1)}=\left[\begin{array}{cccc}
-2 & 2 / 3 & 0 & 0 \\
16 / 3 & 0 & 2 / 3 & -2 / 3 \\
-2 & -14 / 3 & 16 / 3 & 16 / 3 \\
-16 / 3 & 0 & 2 / 3 & -2 / 3
\end{array}\right] .
$$


Il en résulte que la différentielle de $f$ au point $(-1,1,1,1)$ est de rang maximum. On peut vérifier que $f(-1,1,1,1)=(12,0,24,0,12)$ et que si $(\alpha, \lambda, \mu, \nu)$ est admissible et vérifie $f(\alpha, \lambda, \mu, \nu)=(12,0,24,0,12)$ alors $(\alpha, \lambda, \mu, \nu)=(-1,1,1,1)$. On remarque que $\Psi^{-1}(12$ : $0: 24: 0: 12)=\{(1: 0: 1)\}$ et que la différentielle de $\Psi$ en (1: 0 : 1 ) est de rang 3 . Dans la carte affine $\tau_{1}=1$, l'application $\Psi$ s'écrit $\widetilde{\Psi}:(B, C) \mapsto$ $\left(2 B, B^{2}+2 C, 2 B C, C^{2}\right)$. Le plan tangent à $f^{-1}(\Theta)$ au point $(-1,1,1,1)$ est l'image de

$$
\operatorname{Jac}(f)_{(-1,1,1,1)}^{-1} \operatorname{Jac}(\widetilde{\Psi})_{(0,1)}=\left[\begin{array}{cc}
0 & 0 \\
3 & 0 \\
\frac{3}{2} & \frac{3}{2} \\
\frac{3}{2} & -\frac{3}{2}
\end{array}\right]
$$

Par suite sur $f^{-1}(\Theta)$ au voisinage de $(-1,1,1,1)$ et à l'ordre 1 on a

$$
\begin{aligned}
& \alpha=-1, \quad \lambda=1+3 u, \quad \mu=1+\frac{3}{2}(u+v), \\
& \nu=1+\frac{3}{2}(u-v),
\end{aligned}
$$

ou encore $\alpha=-1$, et $\nu-1-\lambda+\mu=0$. En particulier, on en déduit l'énoncé suivant.

Théorème 5.19. L'espace des paramètres $(\alpha, \lambda, \mu, \nu)$ admissibles pour lesquels est associée à $\mathcal{F}(\alpha ; \lambda, \mu, \nu)$ une trivolution est localement au point $(-1,1,1,1)$ une surface lisse.

La trace de cette surface est précisée sur la section hyperplane $\alpha=-1$ par la proposition qui suit.

Proposition 5.20. On peut associer une trivolution $\grave{a}$ $\mathcal{F}(-1 ; \lambda, \mu, \nu)$ si et seulement si le quadruplet admissible $(-1, \lambda, \mu, \nu)$ satisfait l'une des propriétés suivantes

(a) $(4-\nu) \lambda-\nu(2 \nu+1)=0$ et $\mu=\nu$;

(b) $\lambda=1$ et $\mu+\nu-4 \mu \nu+2=0$.

Remarque 5.21. On remarque que $(-1,1,1,1)$ vérifie les conditions (a) et (b).

Remarque 5.22. On passe de la condition (a) à la condition (b) par $(x, y) \mapsto(x+y, y-x)$, la non homogénéité apparente résultant du fait qu'on a normalisé à 1 l'exposant de $x$.

Démonstration. Le quadruplet $(-1, \lambda, \mu, \nu)$ est supposé admissible. Quitte à poser $u=\mu+\nu$ et $w=\mu-\nu$ les $r_{i}$ s'écrivent

$$
\begin{aligned}
r_{1}= & \lambda^{2}\left(4 u+4+w^{2}\right), \\
r_{2}= & 2 \lambda w\left(-u-2+\lambda u-w^{2}+u^{2}+2 \lambda\right), \\
r_{3}= & u^{2}-2 \lambda u w^{2}-8 \lambda^{2}+2 u^{3}-2 u w^{2}-2 \lambda u^{2}-2 \lambda w^{2} \\
& -4 \lambda u+2 \lambda u^{3}-4 \lambda^{2} u+\lambda^{2} u^{2}+\left(u^{2}-w^{2}\right)^{2}, \\
r_{4}= & 2 w\left(u-\lambda u-w^{2}+u^{2}+2 \lambda-2 \lambda^{2}\right), \\
r_{5}= & 4 \lambda^{2}+4 \lambda u+w^{2} .
\end{aligned}
$$

Remarquons que $\lambda+\mu+\nu+1 \neq 0$ se réécrit $\lambda+u+1 \neq$ 0 . Cette présentation, bien qu'asymétrique, permet en fait d"alléger" les calculs qui vont suivre.

Pour que la condition (i) soit vérifiée il faut que $r_{5}$ soit nul autrement dit que $u=-\frac{4 \lambda^{2}+w^{2}}{4 \lambda}$. Alors $r_{4}$ se réécrit

$$
\frac{2 w}{\lambda^{2}}\left(\lambda-\frac{w}{2}\right)\left(\lambda+\frac{w}{2}\right)\left(\lambda-\frac{w^{2}}{4}\right) .
$$

Comme le quadruplet $(-1, \lambda, \mu, \nu)$ est admissible (c'està-dire $\lambda \mu \nu \neq 0$ et $\lambda+\mu+\nu+1 \neq 0$ ), on vérifie que les conditions $r_{4}=0$ et $4 r_{1} r_{3}-r_{2}^{2}=0$ impliquent que $\lambda=1$ et $\mu=\nu=-1 / 2$. Ces valeurs des paramètres satisfont la condition (b). Ceci correspond exactement à l'Exemple 5.18 .

Étudions maintenant la condition (ii) que l'on traduit par $r_{5} \neq 0, r_{4}=r_{2}=r_{3}^{2}-4 r_{1}=0$. Toujours en invoquant l'admissibilité on vérifie que les conditions $r_{2}=r_{4}=0$ sont satisfaites pour (1) $w=0$ ou (2) $\lambda(u+2)-w^{2}+u^{2}-u-2=0$ et $u^{2}+u-\lambda u-2 \lambda^{2}+2 \lambda=0$, conditions que nous allons examiner cas par cas.

Si $w$ est nul, i.e. $\mu=\nu$, on vérifie que $r_{3}^{2}-4 r_{1} r_{5}=$ $-u^{3}(u+\lambda+1)^{3}((u-8) \lambda+u(u+1))$. Maintenant l'admissibilité exige la non-nullité de $u$ et $u+\lambda+1$; de sorte que $r_{3}^{2}-4 r_{1} r_{5}=0$ si et seulement si $(u-8) \lambda+$ $u(u+1)=0$. Finalement on obtient

$$
\mu=\nu \quad \text { et } \quad(4-\nu) \lambda-\nu(2 \nu+1)=0 .
$$

On constate ensuite que l'éventualité (2) ne produit pas de nouvelles solutions admissibles.

Pour finir traitons la condition (iii). On constate que $r_{4}^{2} r_{1}-r_{5} r_{2}^{2}=16 \lambda^{2} w^{2}(\lambda-1)(u+w)(u-w)(\lambda+u+1)^{3}$.

Le quadruplet $(-1, \lambda, \mu, \nu)$ est admissible et $r_{4}$ est non nul donc $w$ aussi. Il s'en suit que $\lambda$ vaut 1 ; alors $4 r_{3} r_{4} r_{5}-$ $r_{4}^{3}-8 r_{2} r_{5}^{2}$ se réécrit

$$
32 w(u+2)^{3}(u+w)(u-w)\left(u^{2}-w^{2}-u-2\right)
$$

ou encore

$$
128(\mu-\nu)(\mu+\nu+2)^{3} \mu \nu(4 \mu \nu-\mu-\nu-2) .
$$


Puisque $\mu, \nu$ et $\mu+\nu+\lambda+1$ sont supposés non nuls,

$$
4 r_{3} r_{4} r_{5}-r_{4}^{3}-8 r_{2} r_{5}^{2}=0
$$

si et seulement si

$$
\mu+\nu-4 \mu \nu+2=0 .
$$

Décrivons précisément la première famille de la Proposition 5.20, la seconde s'en déduit (Remarques 5.21 et 5.22). Le feuilletage $\mathcal{F}$ est alors défini par le champ

$$
\frac{\nu x\left((2 \nu+1) x^{2}-9 y^{2}\right)}{4-\nu} \frac{\partial}{\partial x}-y\left((2 \nu+1) x^{2}-y^{2}\right) \frac{\partial}{\partial y} .
$$

On vérifie que $\Delta(P)$ est un carré

$$
\begin{aligned}
\Delta(P)= & \frac{1024 \nu^{2}(2 \nu+1)^{5}}{(\nu-4)^{6}} x^{4} y^{4}\left(x^{2}-y^{2}\right)^{4} \\
& \times\left((2 \nu+1) x^{2}+3 y^{2}\right)^{2} .
\end{aligned}
$$

Choisissons une racine $\widetilde{\nu}$ de $2 \nu+1$. À $\mathcal{F}$ est associée une trivolution $\mathcal{T}_{\mathcal{F}}$ de la forme $\left(\frac{U_{1}}{W U_{2}}, \frac{V_{1}}{W V_{2}}\right)$ avec

$$
\begin{aligned}
& U_{1}=4 x y(\nu \widetilde{\nu}(2 \nu+1)^{2} x^{5} \\
&-\left(12 \nu^{3}+28 \nu^{2}+19 \nu+4\right) x^{4} y \\
&+2 \nu \widetilde{\nu}\left(\nu-2 \nu^{2}+1\right) x^{3} y^{2} \\
&+2\left(6 \nu^{3}+13 \nu^{2}+13 \nu+4\right) x^{2} y^{3} \\
&\left.-3 \nu \widetilde{\nu}(2 \nu+1) x y^{4}+\left(2 \nu^{2}-7 \nu-4\right) y^{5}\right), \\
& V_{1}=4 x y(\nu\left.+6 \nu+12 \nu^{2}+8 \nu^{4}\right) x^{5} \\
&+\widetilde{\nu}\left(4 \nu^{3}-12 \nu^{2}-15 \nu-4\right) x^{4} y \\
&+2 \widetilde{\nu}\left(9 \nu^{2}-3 \nu-4-2 \nu^{3}\right) x^{2} y^{3} \\
&-2\left(45 \nu^{2}+16 \nu^{3}+4 \nu^{4}+8+35 \nu\right) x^{3} y^{2} \\
&+\left(20 \nu^{3}+16+69 \nu+84 \nu^{2}\right) x y^{4} \\
&\left.+3 \widetilde{\nu}\left(4-2 \nu^{2}+7 \nu\right) y^{5}\right), \\
& \\
& W=\nu^{2}(2 \nu+1)^{2} x^{4}-2\left(2 \nu^{3}+25 \nu^{2}+28 \nu+8\right) x^{2} y^{2} \\
&+(\nu-4)^{2} y^{4}, \\
& U_{2}=(2 \nu+1) x^{2}-y^{2}, \quad V_{2}=(2 \nu+1) x^{2}-9 y^{2} .
\end{aligned}
$$

\section{CONCLUSION}

Les exemples qui précèdent soulèvent plus de problèmes qu'ils n'en résolvent et on peut dégager un certain nombre de questions. En premier lieu on peut se demander quels sont les feuilletages associés aux involutions de Bertini; ont-ils des propriétés (dynamiques) spéciales? Dans un autre ordre d'idées nous avons plusieurs fois mentionné la construction de 3 -tissus associés à certains feuilletages produisant des trivolutions. Le fait que ces 3 -tissus soient hexagonaux est-il un fait anecdotique? Enfin il serait intéressant de décrire complètement la variété des feuilletages de degré 3 qui produisent des trivolutions. En particulier quel est son degré? Est-elle irréductible? Rationnelle?

\section{REMERCIEMENTS}

Dans [Williams 20] on trouve le passage suivant: "However, Professor H. S. White, to whom this paper was refered, pointed out the subject had been well covered by Geiser and Milinowski". Nous remercions le referee qui nous a indiqué cette littérature ancienne et dont le commentaire était proche de celui de White.

\section{REFERENCES}

[Bayle and Beauville 00] L. Bayle and A. Beauville. "Birational involutions of $\mathbf{P}^{2}$." Asian J. Math. 4:1 (2000), 11-17.

[Beauville 80] A. Beauville. "Géométrie des tissus [d'après S. S. Chern et P. A. Griffiths]." In Séminaire Bourbaki (1978/79), Lecture Notes in Math. 770, pp. 103-119. Berlin: Springer, 1980.

[Bertini 77] E. Bertini. "Ricerche sulle trasformazioni univoche involutorie nel piano." Annali di Mat. 8 (1877), 244286 .

[Camacho and de Figueiredo 01] C. Camacho and L. H. de Figueiredo. "The Dynamics of the Jouanolou Foliation on the Complex Projective 2-Space." Ergodic Theory Dynam. Systems 21:3 (2001), 757-766.

[Campillo and Olivares 00] A. Campillo and J. Olivares. "Assigned Base Conditions and Geometry of Foliations on the Projective Plane." In Singularities-Sapporo 1998, Adv. Stud. Pure Math. 29, pp. 97-113. Tokyo: Kinokuniya, 2000 .

[Cerveau and Lins Neto 91] D. Cerveau and A. Lins Neto. "Holomorphic Foliations in $\mathrm{CP}(2)$ Having an Invariant Algebraic Curve." Ann. Inst. Fourier (Grenoble) 41:4 (1991), 883-903.

[Cerveau et al. 09] D. Cerveau, J. Déserti, D. Garba Belko, and R. Meziani. "Géométrie classique de certains feuilletages de degré deux." Bull. Braz. Math. Soc. (N.S.) 41:2 (2010), 161-198.

[de Fernex 04] T. de Fernex. "On Planar Cremona Maps of Prime Order." Nagoya Math. J. 174 (2004), 1-28.

[Dolgachev and Iskovskikh 10] Igor V. Dolgachev and Vasily A. Iskovskikh. "Finite Subgroups of the Plane Cremona Group." In Algebra, Arithmetic, and Geometry: In Honor of Yu. I. Manin., Vol. 1, pp. 443-548, Progr. Math., 269, Boston: Birkhäuser Boston, 2009. 
[Gómez-Mont and Kempf 89] X. Gómez-Mont and G. Kempf. "Stability of Meromorphic Vector Fields in Projective Spaces." Comment. Math. Helv. 64:3 (1989), 462-473.

[Jouanolou 79] J. P. Jouanolou. Équations de Pfaff algébriques, Lecture Notes in Mathematics 708. Berlin: Springer, 1979.
[Pereira 01] J. V. Pereira. "Vector Fields, Invariant Varieties and Linear Systems." Ann. Inst. Fourier (Grenoble) 51:5 (2001), 1385-1405.

[Williams 20] A. R. Williams. "On a Birational Transformation Connected with a Pencil of Cubics." University of California Publications in Mathematics, 20:10 (1920), 211222.

Dominique Cerveau, Membre de l'Institut Universitaire de France. IRMAR, UMR 6625 du CNRS, Université de Rennes 1, 35042 Rennes, France. (dominique.cerveau@univ-rennes1.fr)

Julie Déserti,Institut de Mathématiques de Jussieu, Université Paris 7, Projet Géométrie et Dynamique, Site Chevaleret, Case 7012, 75205 Paris Cedex 13, France. (deserti@math.jussieu.fr)

Received November 6, 2009; accepted April 14, 2010. 